\title{
Review of the Canadian Eustrophinae (Coleoptera, Tetratomidae)
}

\author{
Darren A. Pollock \\ Department of Biology, Eastern New Mexico University, Portales NM USA \\ Corresponding author: Darren A. Pollock (darren.pollock@enmu.edu)
}

Academiceditor: ChristopherMajka| Received20September2008|Accepted 14October2008|Published 17October 2008

Citation: Pollock DA (2008) Review of the Canadian Eustrophinae (Coleoptera, Tetratomidae). In: Majka CG, Klimaszewski J (Eds) Biodiversity, Biosystematics, and Ecology of Canadian Coleoptera. ZooKeys 2: 261-290. doi: $10.3897 /$ zookeys.2.30

\begin{abstract}
Currently, the Canadian fauna of Eustrophinae consists of 7 species in 5 genera, as follows: Pseudoholostrophus impressicollis (LeConte), P. discolor (Horn); Holostrophus bifasciatus (Say); Eustrophus tomentosus Say; Eustrophopsis bicolor (Fabricius), E. confinis (LeConte); Synstrophus repandus (Horn). None of these 7 species is restricted to Canada; each has a wider distribution in the United States. Each species (adult stage only) is diagnosed and described with selected morphological features illustrated. A key to Nearctic genera, and Canadian species of Eustrophinae is presented, as well as a checklist of all Nearctic species of the subfamily. Lectotypes are designated for Holostrophus discolor Horn and Eustrophus confinis LeConte. Neotypes are designated for Eustrophus bifasciatus Say and Eustrophus tomentosus Say. The Canadian distribution of each species is mapped in detail, as well as a general indication of distribution in the United States. Aspects of the natural history of all species, where known, are included.
\end{abstract}

\section{Keywords}

Tetratomidae, Canada, distribution, natural history, faunistics, identification key

\section{Introduction}

The Eustrophinae are a relatively small, structurally and ecologically homogenous group of tenebrionoid beetles. Like many other taxa of the superfamily, these beetles have not had a stable family placement until recently. This instability is due at least partly to the over-reliance on adult characters, which suggested to some authors a relationship between the Eustrophinae and Melandryidae (e.g. Arnett 1968). Other non-North American authors (e.g. Crowson 1964; Hayashi 1975; Viedma 1971) suggested a relationship between Eustrophinae and Tetratomidae, based on both adult and larval characteristics. 
Indeed, due to the popularity of Arnett (1968), most North American coleopterists were unfamiliar with the family Tetratomidae, since Arnett chose to incorporate its constituents into a very broad concept of Melandryidae. In the most recent comprehensive family/subfamily-level classification of Coleoptera, Lawrence and Newton (1995) placed the Eustrophinae again within Melandryidae. However, the eustrophines seem to have found a stable family placement within the Tetratomidae, as outlined by Nikitsky (1998). Earlier, Chantal (1985) also placed the eustrophines in Tetratomidae, without giving any detailed explanation. Nikitsky (1998) treated the genera of Tetratomidae worldwide, and combined larval and adult characters into the classification. He also created several new taxa within the subfamily, notably the new tribe Holostrophini and a new subgenus Holostrophinus, and synonymized the name Eustrophinus Seidlitz with Eustrophopsis, the latter of which was formerly restricted to mainly tropical species with a notched prosternal process. No phylogenetic analysis accompanied the treatment of Nikitsky (1998) but placement within Tetratomidae seems well supported.

Like most groups of Coleoptera, relatively little is known about the ecology and habits of members of Eustrophinae, although they are associated with various fungi found in and on coarse woody debris in forest habitats. Most fungal host records are within the Polyporales (e.g. Polyporus, Fomes, Trametes, Meripilus, Panus, Spongipellus, and Laetiporus), with several genera of Agaricales also (e.g. Pleurotus and Omphalotus). Specimens have also been taken from fungi on isolated trees, for example, in urban areas. Host records and habitat associations are given in Chantal (1985), Lawrence (1991) and Majka and Pollock (2006). When observed and/or collected in situ, adult eustrophines are most often found on fruiting bodies of the fungi, or adjacent wood. Larvae are often found deeper within the substrate, where there is a mixture of fungal hyphae and rotting wood. Adult eustrophines are uncommonly collected in long series, except when they are trapped (e.g. in Lindgren funnel traps) or sought at night on fungusy wood. The fact that there are gaps in the distributions of species such as Eustrophus tomentosus Say and Synstrophus repandus (Horn) may be due more to lack of effective collecting methods than to unsuitable habitat or other barriers. Details of natural history are given below, for individual species; most data are taken directly from specimen labels.

There is presently no comprehensive treatment of any genus of Eustrophinae or of the entire subfamily; for North American components of the group, all that has been published are checklists (e.g. LeSage 1991) or keys to genera (e.g. Arnett 1968; Young and Pollock 2002). Nothing specific has been published regarding the Canadian eustrophines, although Chantal's (1985) "Tetratomidae of Quebec" included six of the seven species of Eustrophinae found in Canada. In fact, southern Quebec is the area of highest diversity of Canadian Eustrophinae. In this work, species are keyed, described briefly, and distributions mapped. Also, data on biology and natural history were presented for each species. The present study, while overlapping considerably at the species level, is an extension of the excellent work done by Chantal (1985), as it enlarges the coverage to the entire country, and also updates the taxonomy, notably at the genus and subgenus levels.

No species of Eustrophinae are restricted to Canada; rather, the Canadian distribution of each represents the northern range limit of an otherwise fairly widespread 
U.S. distribution. For example, Pseudoholostrophus discolor (Horn) is known from two localities in Canada, while others, such as Eustrophopsis confinis (LeConte) are relatively more widespread across the country. One species, P. impressicollis (LeConte), is restricted to extreme western Canada. Three species - Eustrophopsis confinis, Eustrophus tomentosus and Synstrophus repandus - are known from British Columbia to Quebec and/or the Maritime provinces, with gaps in Alberta and Saskatchewan (a single record of $E$. confinis is known from Saskatchewan). As mentioned above, these gaps perhaps have more to do with incomplete collecting, rather than some biogeographic artifact. However, there have been, and still are, very keen beetle collectors in Alberta and Saskatchewan. As part of a paper on saproxylic beetles, Majka and Pollock (2006) provided new Canadian Maritime records for several species.

\section{Material and methods}

Adult specimens of Eustrophinae collected in Canada were borrowed from the collections below; the collection abbreviations are used in the locality lists. Several thousand specimens of U.S. -collected eustrophines were also examined (as part of a larger study of Nearctic Eustrophinae); these specimens were used to construct the inset maps, showing US distributions for each species known from Canada. Specimens mentioned by Chantal (1985) were not examined in this study; however, the localities mentioned in that work were used in the distribution maps.
AMNH American Museum of Natural History, New York, NY
CARR J.B. and A. Carr Collection (now part of CNC)
CGMC Chris G. Majka Collection (private)
CMNC Canadian Museum of Nature, Ottawa, ON
CMNH Carnegie Museum of Natural History, Pittsburgh, PA
CNC Canadian National Collection of Insects, Ottawa, ON
CUIC Cornell University, Ithaca, NY
DAPC Darren A. Pollock Collection (private)
DEBU University of Guelph, Guelph, ON
FMNH Field Museum of Natural History, Chicago, IL
FSCA Florida State Collection of Arthropods, Gainesville, FL
GJHC Gerald Hilchie Collection (private)
JBWM J.B. Wallis Museum of Entomology, Winnipeg, MB
MCZ Museum of Comparative Zoology, Cambridge, MA
PFC Pacific Forestry Centre, Victoria, BC
RBCM Royal British Columbia Museum, Vancouver, BC
ROME Royal Ontario Museum, Toronto, ON
SLC Serge LaPlante Collection (private)
UAIC University of Arizona, Tucson, AZ
UBC University of British Columbia, Vancouver, BC 
USNM National Museum of Natural History, Smithsonian Institution, Washington, DC

UWEM University of Wisconsin, Madison, WI

Standard methods of examination and description of specimens were used. Published lists of localities for Canadian Eustrophinae (e.g. Chantal 1985) were used to construct the distribution maps, but specific locality data are not given under the section "material examined".

Due to the regional aspect of this paper and because it is not meant to be a taxonomic revision, full descriptions are not given for genera or species; rather, descriptions are diagnostic in nature, with emphasis on characteristics that will facilitate rapid determination of species. Complete descriptions (of genera and species) will be published elsewhere, as part of a comprehensive review of the entire subfamily Eustrophinae.

Because of the relative rarity of specific life history data, all known information is given for each species, even if taken from specimens collected outside of Canada. The geographic origin of such extralimital information is indicated by the standardized, two-letter postal abbreviation following each datum.

In the descriptions, $\mathrm{TL}=$ length from anterior margin of pronotum, to elytral apex along midline. The length of the head was not used, since the head is deflexed and therefore does not contribute to body length. GEW = greatest width across both elytra.

For type specimens, complete verbatim label data are given, enclosed within quotation marks; individual labels are separated by a slash (/).

Figures 1-14 were taken with a JVC digital camera KY-F775U attached to a Leica Z16 APO stereoscope. Images were then modified using Auto-Montage Pro version 5.01.0005. Figures 15-16 were taken through a Leica MZ95 stereoscope, using a Nikon CoolPix digital camera; they were not modified further. Figures 17-25 were produced using a camera lucida attached to a Wild stereoscope.

\section{Identification key to Nearctic genera and Canadian species of Eustrophinae}

1 Outer faces of meso- and metathoracic tibiae with numerous oblique, comblike ridges (as in Fig. 16) ........................................................................... 2

- $\quad$ Outer faces of meso- and metathoracic tibiae with scattered spines but without oblique ridges (Fig. 15) ................................................................... 4

2(1) Prothoracic episterna with distinct, transverse suture; dorsal surface dark piceous to black, without brownish iridescence (Eustrophopsis Champion) ..... 3

- $\quad$ Prothoracic episterna without transverse suture; dorsal surface distinctly brown, slightly iridescent .................................... Eustrophus tomentosus Say

3(2) Innermost margins of eyes widely separated by distance greater than length of first antennomere (Fig. 13); entire body dark brown to black, without distinctly contrasting lighter color ventrally or on antennae 

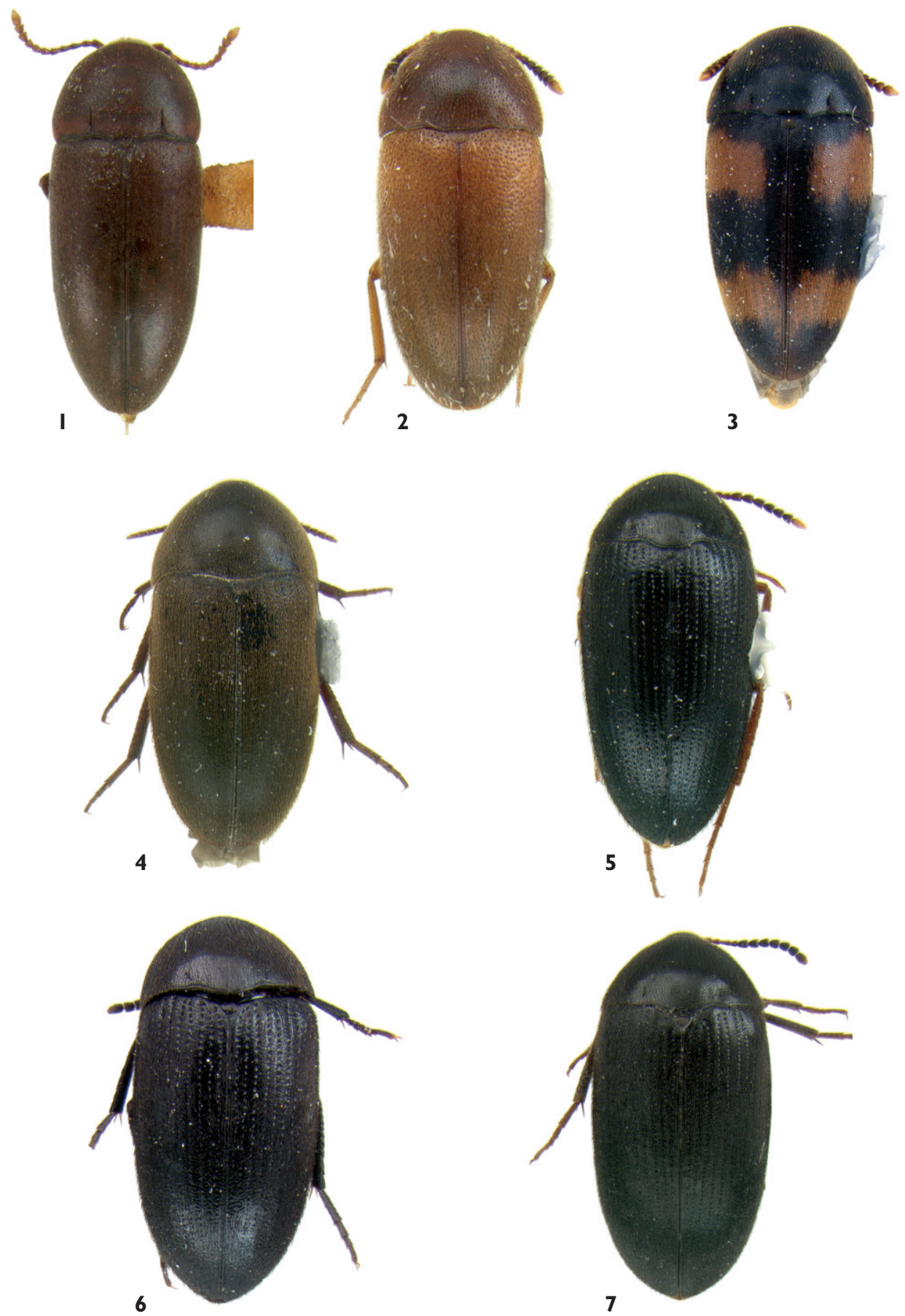

Figs I-7. Dorsal habitus of Canadian eustrophines. Fig. 1. Pseudoholostrophus impressicollis, TL = 4.3 $\mathrm{mm}$; Fig. 2. Pseudoholostrophus discolor, $\mathrm{TL}=4.8 \mathrm{~mm}$; Fig. 3. Holostrophus bifasciatus, $\mathrm{TL}=5.8 \mathrm{~mm}$; Fig. 4. Eustrophus tomentosus, $\mathrm{TL}=4.8 \mathrm{~mm}$; Fig. 5. Eustrophopsis bicolor, $\mathrm{TL}=5.6 \mathrm{~mm}$; Fig. 6. Eustrophopsis confinis, $\mathrm{TL}=5.4 \mathrm{~mm}$; Fig. 7. Synstrophus repandus, $\mathrm{TL}=6.8 \mathrm{~mm}$. 

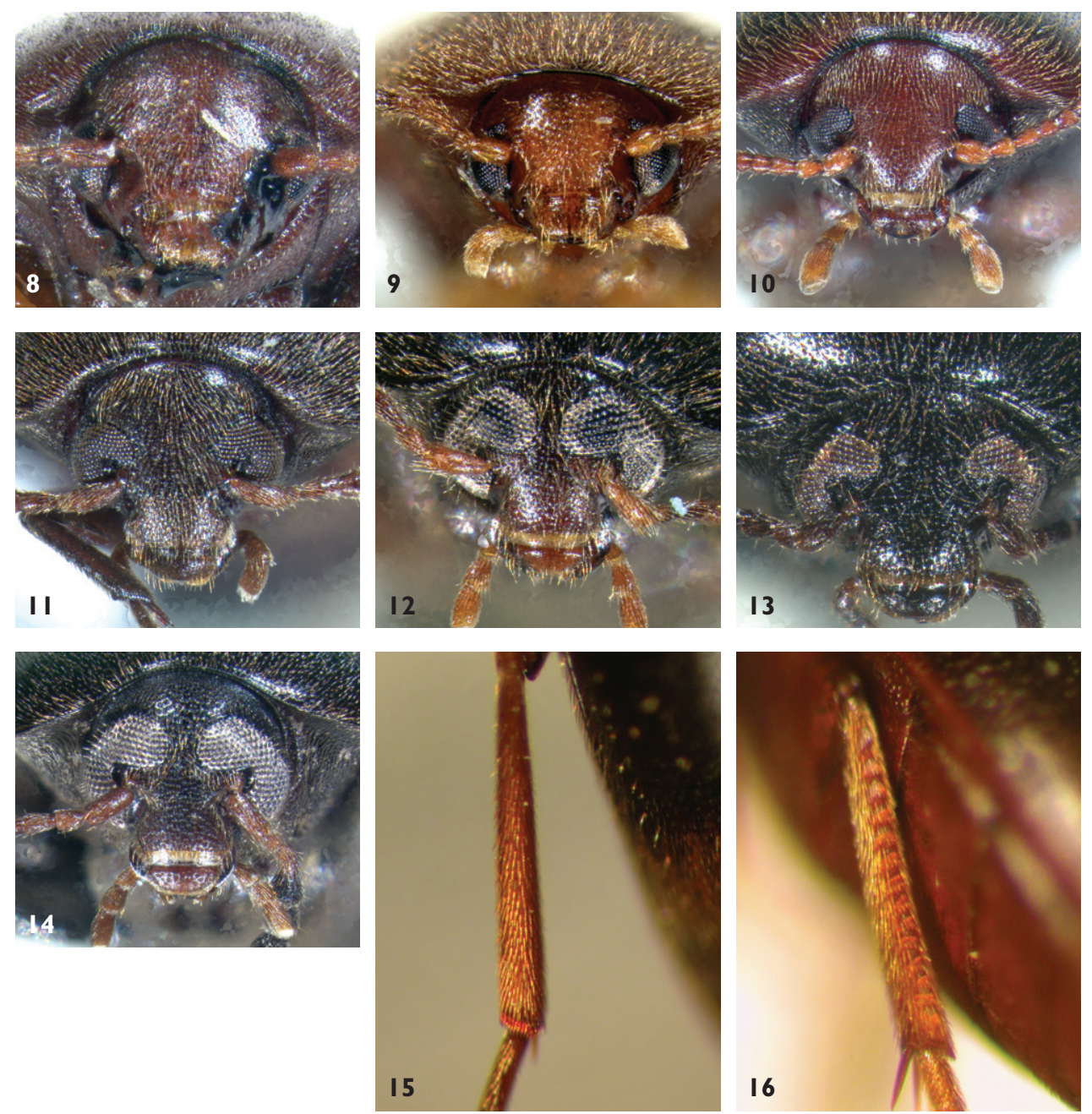

Figs 8-16. Features of Canadian eustrophines. Figs. 8-14. Frontal view of head (same specimens as in Figs. 1-7). Fig. 8. Pseudoholostrophus impressicollis; Fig. 9. Pseudoholostrophus discolor; Fig. 10. Holostrophus bifasciatus; Fig. 11. Eustrophus tomentosus; Fig. 12. Eustrophopsis bicolor; Fig. 13. Eustrophopsis confinis; Fig. 14. Synstrophus repandus. Figs. 15-16. Hind tibia. Fig. 15. H. bifasciatus; Fig. 16. E. tomentosus.

- $\quad$ Innermost margins of eyes more narrowly separated (Fig. 12), in some specimens virtually contiguous; if slightly separated, then gap less than length of first antennomere; body dark dorsally, with legs, much of venter and antennomere 11 distinctly contrasting.................. Eustrophopsis bicolor (Fabricius)

4(1) Prosternal process narrowed distally, not extending posteriad of prothoracic coxae; elytral punctation coarse, forming distinct striae (Fig. 7)

Synstrophus repandus (Horn)

- $\quad$ Prosternal process widened distally, separating prothoracic coxae; elytral punctation fine to coarse, not forming distinct striae (Holostrophini)........ 5 
5(4) Eyes distinctly emarginate (Fig. 10); prosternal process longer, protruding behind posterior edge of prothoracic coxae; elytra dark in color, with 4 subquadrate, lighter colored maculae (Fig. 3) ......... Holostrophus bifasciatus (Say)

- $\quad$ Eyes only indistinctly emarginate (Figs 8-9); prosternal process at most slightly projecting behind posterior edge of prothoracic coxae; elytra uniformly rufous to piceous, without distinctly delimited lighter areas (Pseudoholostrophus Nikitsky)

6(5) Dorsal surface finely punctate (Fig. 1), setae short and adpressed against surface; impressions along posterior pronotal margin linear, parallel, distinctly impressed; anterior margin of pronotum not elevated shelf-like above level of head; known only from western North America (in Canada, only British Columbia) Pseudoholostrophus impressicollis (LeConte)

- $\quad$ Dorsal surface more coarsely punctate (Fig. 2), setae more distinct and at least partly erect; impressions along posterior pronotal margin indistinct; anterior margin of pronotum distinctly elevated above level of head; known only from eastern North America (in Canada, only southern Quebec)

Pseudoholostrophus discolor (Horn)
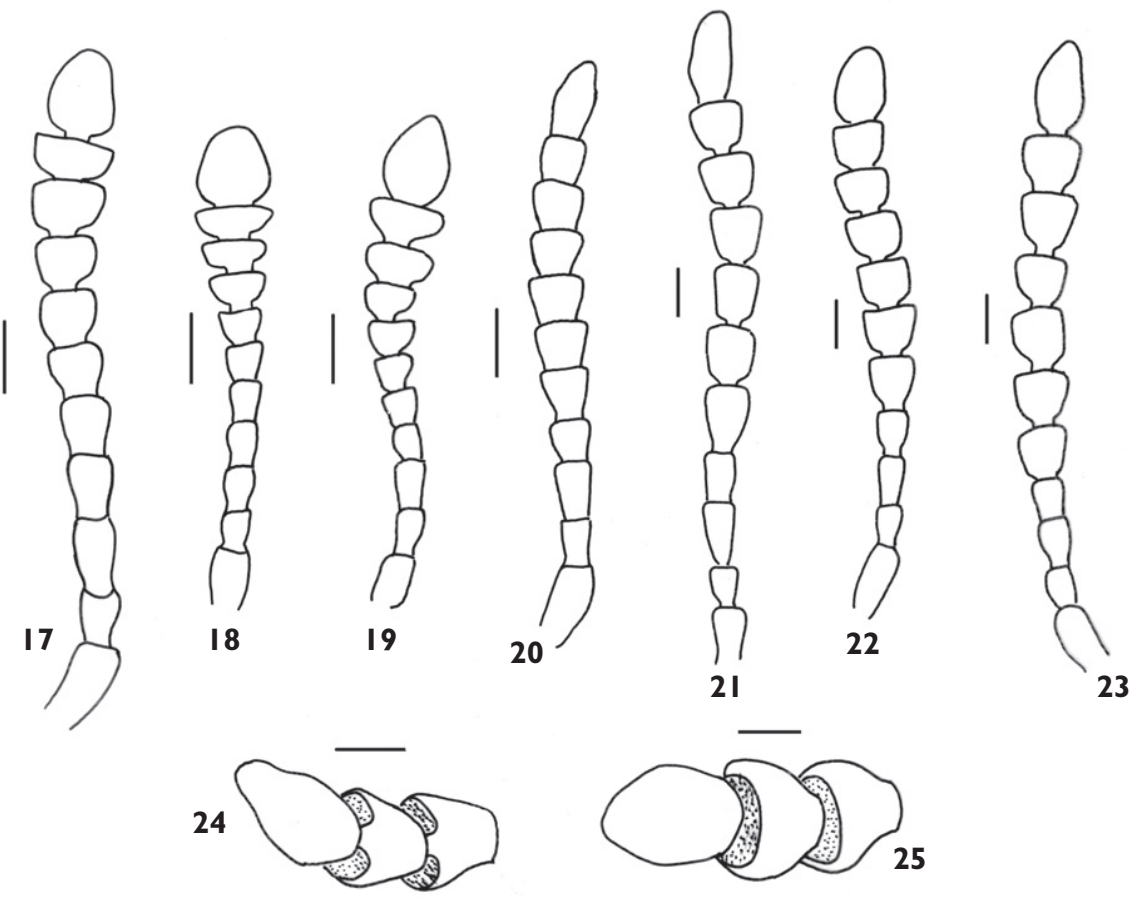

Figs 17-25. Antennae of Canadian eustrophines. Fig. 17. Pseudoholostrophus impressicollis; Fig. 18. Pseudoholostrophus discolor; Fig. 19. Holostrophus bifasciatus; Fig. 20. Eustrophus tomentosus; Fig. 21. Eustrophopsis bicolor; Fig. 22. Eustrophopsis confinis; Fig. 23. Synstrophus repandus; Fig. 24. E. tomentosus, antennomeres 9-11 (sensillar field indicated by stippling); Fig. 25. S. repandus, antennomeres 9-11 (sensillar field indicated by stippling). Scale bar = 0.25 mm (Figs. 17-23); $0.1 \mathrm{~mm}$ (Figs. 24-25). 


\section{Species accounts}

Tribe Holostrophini Nikitsky, 1998

\section{Pseudoholostrophus Nikitsky, 1983}

Pseudoholostrophus Nikitsky, 1983: 37.-Type species: Hallomenus klapperichi Pic, 1954 (orig. des.); Nikitsky, 1998: 40; Young and Pollock, 2002: 416.

This genus was described by Nikitsky (1983) on the basis of examination of Hallomenus klapperichi Pic, which is now included in, and type species of Pseudoholostrophus. According to Nikitsky (1998), Pseudoholostrophus differs from Holostrophus in its smaller and more weakly emarginate eyes, and the prosternal process not extending behind the posterior edge of the procoxae. There are four species in Pseudoholostrophus (Nikitsky, 1998), two of which are Nearctic (including Canada) in distribution. The other two species, $P$. klapperichi (Pic) and $P$. chinensis Nikitsky, are known from China. Nikitsky (1998) divided the genus into two subgenera; all species other than $P$. discolor (Horn) are placed in the nominate subgenus. Holostrophus discolor (Horn) was transferred to Pseudoholostrophus (Holostrophinus) by Nikitsky (1998).

Subgenus Pseudoholostrophus Nikitsky, 1983

Pseudoholostrophus (Pseudoholostrophus) impressicollis (LeConte, 1874)

(Figs 1, 8, 17, 26)

Eustrophus impressicollis LeConte, 1874: 69; Horn, 1888: 36; Leng, 1920: 238; Csiki, 1924: 10; Poole and Gentili, 1996: 299; LeSage, 1991: 246; Hatch, 1965: 67, Plate IX, fig. 1; Nikitsky, 1998: 47, Plate 7 figs 9-11; Young and Pollock, 2002: 416. Lectotype, sex unknown, labelled "Vanc. / Type 4781 / Eu. impressicollis Lec.", in MCZ.

Diagnosis This distinctive species can be separated from all Nearctic species of Eustrophinae by the combination of the following characteristics: uniform red-brown color, short, inconspicuous dorsal pubescence, widely separated eyes, and smooth meso- and metatibiae.

Description TL 6.0-6.2 mm; GEW 2.1-2.7 mm. Body (Fig. 1) elongate oval, moderately parallel sided. Dorsal and ventral color uniformly dark rufous, including antennae and legs; dorsal pubescence very short, inconspicuous (Fig. 1); eyes (Fig. 8) widely separated ( $>3 \mathrm{x}$ length of first antennomere), inner margin of eye slightly emarginate; antennae (Fig. 17) relatively short, antennomeres 7-10 distinctly widened; antennomere 7 triangular, 8-10 wider than long; antennal sensilla completely annular (as in Fig. 25); last maxillary palpomere distinctly widened, securiform; prosternal process elongate, spatulate distally extended past posterior margin of procoxae, bent dorsally at distal end; prothoracic episternal suture absent; elytral punctation fine, punctures 
not arranged in longitudinal striae; meso- and metatibiae with scattered short spines, without oblique ridges (as in Fig. 15).

Distribution (Fig. 26) This species is restricted to extreme western North America. In Canada, all known records are from Vancouver Island and the adjacent mainland of British Columbia, north to the Queen Charlotte Islands. This is seemingly one of the rarest, or most infrequently collected, species of Nearctic Eustrophinae; most localities are represented by a single specimen. US distribution: WA (Hatch 1965), OR, CA.

Natural history Little is known regarding the habits of $P$. impressicollis. Label data indicate the following: under bark of pine; in rotten log; fungus.

Material examined Specimens were examined from the following Canadian localities: BRITISH COLUMBIA: Gabriola, 2.VI.1994, BF \& JL Carr, lot 7, (CARR, 1); Qualicum, 16.IX.1962, BF \& JL Carr, lot 1, (CARR, 1); Vancouver Island, Kye Bay, nr. Comox, 5 m, 2.VII.1980, H. \& A. Howden, (CMNC, 1); Queen Charlotte Islands, Laskeek Bay, Haswell Island, 2-22.VI.2000, 5251'42”N, 13141'06”W, Allombert, Sylvain, (RBCM, 1).

Subgenus Holostrophinus Nikitsky, 1998

Pseudobolostrophus (Holostrophinus) discolor (Horn, 1888)

(Figs 2, 9, 18, 26)

Holostrophus discolor Horn, 1888: 36.-_Leng, 1920: 238; Csiki, 1924: 10; Poole and Gentili, 1996: 299; LeSage, 1991: 246; Nikitsky, 1998: 43, Plate 7 figs 12-15; Young and Pollock, 2002: 416. Lectotype (here designated), sex unknown, labelled "Va. / Henry Ulke Beetle Coll. CMNH Acc. No. 1645 / Holostrophus discolor Horn / LECTOTYPE Eustrophus discolor Horn design. DA Pollock 2008”, in CMNH. Paralectotype in MCZ.

Diagnosis The smooth meso- and metatibiae, widely separated eyes, conspicuous dorsal pubescence, shelf-like anterior pronotal margin, and eastern distribution serve to separate this species from all other Nearctic eustrophines.

Description TL 3.6-5.7 mm; GEW 1.6-2.4 mm; body (Fig. 1) elongate oval, rather parallel-sided; dorsal color rufous, pronotum in most specimens slightly darker than elytra; some specimens with lighter humeral area on elytra; antennomeres 1-5 rufous, 6-10 rufopiceous, 11 light rufous; venter uniformly red-brown; dorsal pubescence relatively long, conspicuous, with some erect hairs; eyes (Fig. 9) widely separated (space $>3 x$ length of antennomere 1), inner eye margin slightly emarginate; antennae (Fig. 18) relatively short, antennomere 7-11 distinctly widened; antennomere 7 triangular, 8-10 distinctly wider than long; antennal sensilla completely annular (as in Fig. 25); last maxillary palpomere slightly widened, subsecuriform; prosternal process elongate, spatulate distally, extended to past posterior margin of procoxae, bent dorsally at distal end; prothoracic episternal suture absent; elytral punctation relatively coarse, punctures not arranged in longitudinal striae; meso- and metatibiae with scattered short spines, oblique ridges absent (as in Fig. 15). 
Distribution (Fig. 26) This species is known from the fewest Canadian localities of any Nearctic eustrophine, i.e. only one locality in southern Quebec and one in New Brunswick. The species has a broader eastern distribution in the United States. US distribution: IN, MA, MD, NH, PA, RI, TN, VA.

Natural history Given that specimens of $P$. discolor are rarely collected, there are very few data available on its habits or biology. Label data include the following: in polypore fungus; malaise trap (NH); intercept trap (PA). According to Chantal (1985) specimens are known from small polypores on trees, notably cherry (Prunus) and maple (Acer). A single specimen from New Brunswick (CNC) bears a label stating "on or near fleshy polypore fungi on beech log".

Material examined Specimens were examined from the following Canadian localities: QUEBEC: [or Quebec City], 7.VIII.1962, J.-C. Aubé, (DAPC, 1); same locality, 7.VIII.1962, (FMNH, 2); Ste-Foy Quebec, Co., 31.VII.1962, J.-C. Aube, (AMNH, 2). NEW BRUNSWICK: Carelton Co., Wakefield, "Bell Forest Preserve", $46.2200^{\circ} \mathrm{N}$ $67.7231^{\circ} \mathrm{W}, 16 . I X .2006$, R.P. Webster, hardwood forest, on or near fleshy polypore fungi on beech log, (CNC, 1).

Holostrophus Horn, 1888

Holostrophus Horn, 1888: 32.-Type species: Eustrophus bifasciatus Say, 1824 (subs. des.; Nikitsky, 1998: 48); Blatchley, 1910: 1293; Champion, 1915: 139; Leng, 1920: 238; Hatch,

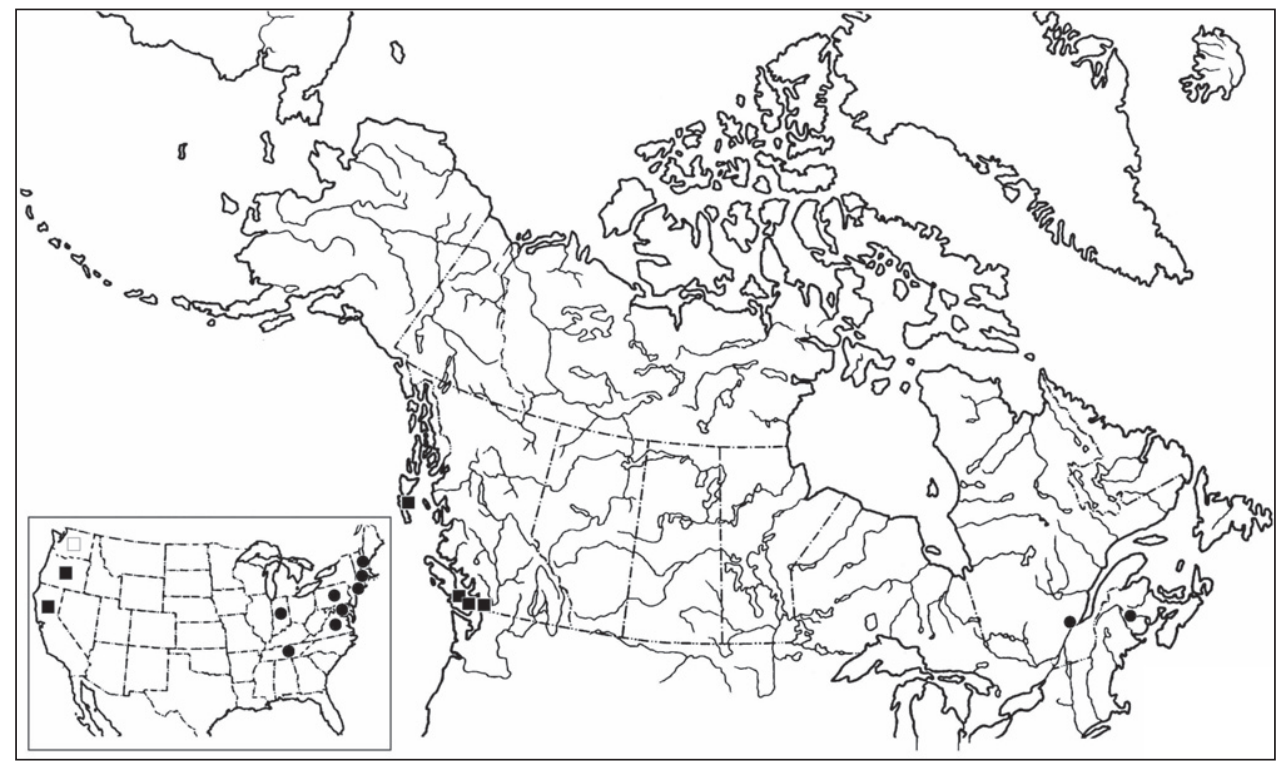

Fig. 26. Canadian distribution of Pseudoholostrophus impressicollis (squares) and $P$. discolor (circles). Inset shows US distribution, by state. Open square indicates state record from Hatch (1965) 
1965: 66; LeSage, 1991: 246; Poole and Gentili, 1996: 299; Nikitsky, 1998: 48; Young and Pollock, 2002: 416.

According to Nikitsky (1998), this genus comprises 17 species worldwide, distributed in the Russian Far East, Korea, China, Japan, the Orient, and Nearctic regions. In North America, there is the single species H. bifasciatus (Say). In further justifying his separation of the genera Pseudoholostrophus and Holostrophus, Nikitsky (1998: 40) stated that "it seems noteworthy that species of Pseudoholostrophus display the elytra either one-color but not black or with a light humeral spot only, or with a clarified diffused transverse fascia in basal part. A more clearly evident reddish-yellow or red spotty pattern of the elytra is characteristic of Holostrophus, not Pseudoholostrophus".

\section{Holostrophus bifasciatus (Say, 1824)}

(Figs 3, 10, 15, 19, 27)

Eustrophus bifasciatus Say, 1824: 282.-Melsheimer, 1853: 143; Blatchley, 1910: 1293; Leng, 1920: 238; Csiki, 1924: 9; Poole and Gentili, 1996: 299; LeSage, 1991: 246; Nikitsky, 1998: plate 9, figs 1-2; Young and Pollock, 2002: 416; Majka and Pollock, 2006: 53. Neotype (here designated), sex unknown, labelled "[faded pink circle] / E. bifasciatus $\underline{\text { Say }}$ 4-maculatus Mels.", in MCZ (LeConte collection).

Eustrophus 4-maculatus Melsheimer, 1846: 58.—Csiki, 1924: 9; Poole and Gentili, 1996: 299; LeSage, 1991: 246.

Diagnosis Holostrophus bifasciatus is the only Nearctic species of Eustrophinae with a distinct, quadrimaculate elytral color pattern.

Description TL 4.1-5.5 mm; GEW 1.9-2.5 mm. Body (Fig. 3) elongate oval, distinctly tapered posteriorly; dorsal color dark rufous to almost black; in most specimens, color of pronotum lighter than color of elytra; elytra with 4 yellow-red, subquadrate maculae: anterior pair near humeri and not attaining suture, posterior pair in apical third of elytra, attaining suture in some specimens; antennomeres uniformly rufous in color, antennomere 11 slightly lighter in color than preceding articles; venter uniformly dark rufous; dorsal pubescence relatively short, inconspicuous; eyes (Fig. 10 ) widely separated (space $>3 x$ length of antennomere 1 ), inner margin of eye moderately deeply emarginate; antennae (Fig. 19) relatively short, antennomeres 7-11 distinctly widened; antennomere 7 triangular, 8-10 distinctly wider than long; antennal sensilla completely annular (as in Fig. 25); last maxillary palpomere slightly widened, subsecuriform; procoxal process elongate, spatulate distally, extended to past posterior margin of procoxae, bent dorsally at distal end; prothoracic episternal suture absent; elytral punctation fine, not arranged in longitudinal striae; meso- and metatibiae with scattered short spines, oblique ridges absent (Fig. 16).

Distribution (Fig. 27) Holostrophus bifasciatus is an eastern species; in Canada, specimens have been collected from west of Lake Superior in northwestern Ontario, 
east to Nova Scotia and Prince Edward Island. Most Canadian specimens are known from southern Ontario and Quebec. The species is very widespread in the eastern half of the United States, almost entirely east of the Mississippi. US distribution: AL, AR, CT, DC, DE, FL, GA, IA, IL, IN, KS, KY, LA, MA, MD, ME, MI, MN, MO, NC, NH, NJ, NY, OH, PA, SC, TN, TX, VA, WI, WV.

Natural history Label data: Laetiporus sulphureus (AR); ex polypore in pine logs (AR); Trametes versicolor (AR); Schizopora paradoxa (AR); BLT (June, AL); ex polypore on cherry tree (AR); under loose pine bark (DE); under pine bark (GA); Malaise trap (March, June, GA); oak log (IL); sugar trap (May, IL); UV light (April, IL); flight trap (Aug-Sept, IL). According to Chantal (1985), specimens of $H$. bifasciatus have been taken under bark of dead Pinus strobus on which was growing polypore fungi, as well as from Polyporus betulinus.

Material examined Specimens were examined from the following Canadian localities: ONTARIO: Arnprior, 26.V.1935, W.J. Brown, (CNC, 12); Essex Co., Windsor Ojibway Prairie, 7.VIII.2001, S. Paiero, Sweeps, (DEBU, 1); Jeannette Creek, 3.VIII.1965, (FSCA, 1); Ottawa, (CNC, 6); same locality, (CUIC, 1); Peel Co., Port Credit, 10.IV.1966, I.M. Smith, (ROME, 1); Tillsonburg, 11.VII.1958, (CNC, 1); Toronto, 20.XI.1895, R.J. Crew, (CUIC, 1); no other data, (DEBU, 3); London, W. Saunders, (DEBU, 1); Ridgeway, (DEBU, 2). QUEBEC: Gatineau, Aylmer, 17.V.1992, Y. Bousquet, in flight, (SLC, 1). PRINCE EDWARD ISLAND: Queens Co., St. Patricks, 25.VI.2003, C.G. Majka, coniferous forest, (CGMC, 2). NOVA SCOTIA: Halifax Co., Pt. Pleasant Park, 15.VII.2001, C.G. Majka, mixed forest, deciduous vegetation, (CGMC, 1).

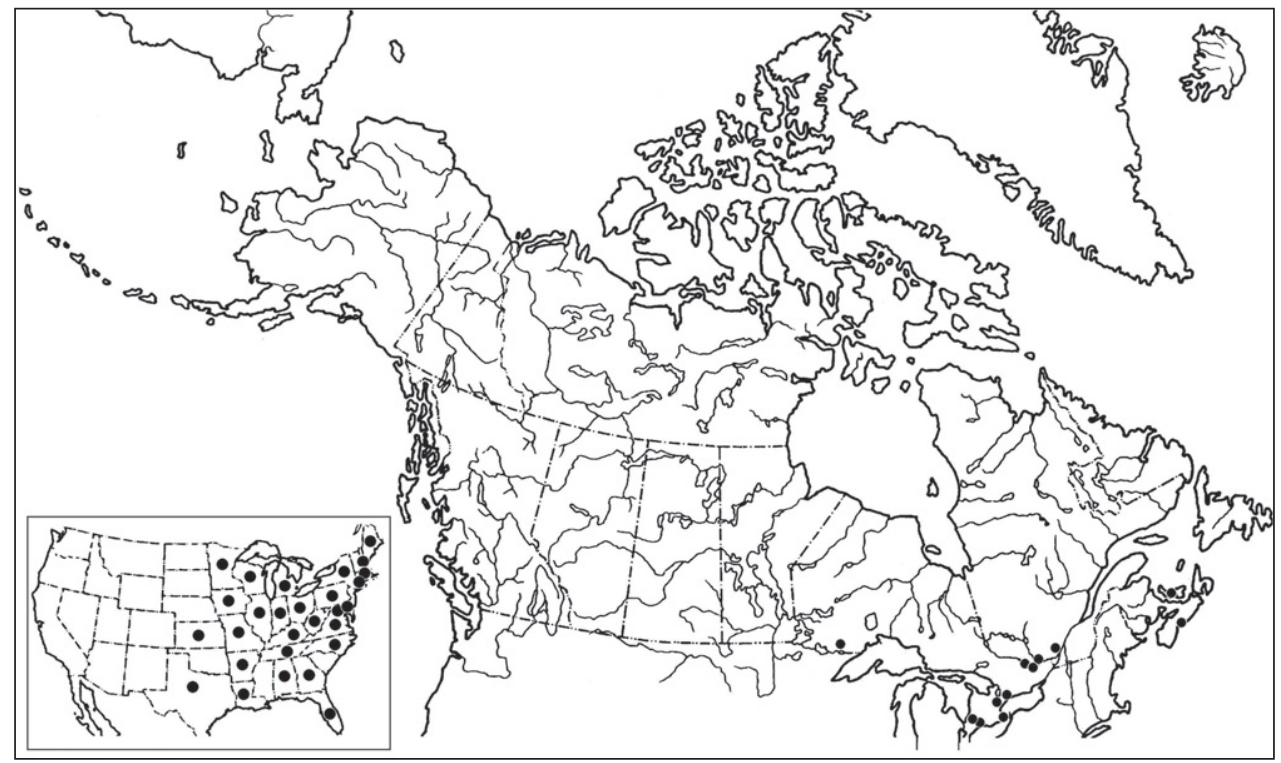

Fig. 27. Canadian distribution of Holostrophus bifasciatus. Inset shows US distribution, by state. 
Tribe Eustrophini Gistel, 1856

Eustrophus Illiger, 1802

EustrophusIlliger, 1802:379.- Type species: Mycetophagusdermestoides Fabricius, 1792 (monotypy); Melsheimer, 1853: 143 (in part); Horn, 1888: 32; Blatchley, 1910: 1292; Champion, 1915: 138; Champion, 1916: 1; Leng, 1920: 238; LeSage, 1991: 246; Poole and Gentili, 1996: 299; Hatch, 1965: 66; Nikitsky, 1998: 54; Young and Pollock, 2002: 416.

This genus comprises four species, distributed in Europe, Russian Far East, Japan, southern China, and North America (Nikitsky, 1998). As this is the oldest genusgroup name available for Eustrophinae, all North American species were at one time included in Eustrophus; now only a single Nearctic species is placed in this genus.

Eustrophus tomentosus Say, 1826

(Figs 4, 11, 16, 20, 24, 28)

Eustrophus tomentosus Say, 1826: 239.—Melsheimer, 1853: 143; Provancher, 1877: 466; Horn, 1888: 35; Blatchley, 1910: 1293; Leng, 1920: 238; Csiki, 1924: 9; Hatch, 1965: 66; Poole and Gentili 1996: 299; LeSage, 1991: 246; Young and Pollock, 2002: 416; Majka and Pollock, 2006: 53. Neotype (here designated), sex unknown, labelled "Ill. / E. tomentosus $\underline{\text { Say. }}$ niger Mels.", in MCZ (LeConte collection).

Mycetophagus niger Melsheimer Catalogue; Melsheimer, 1846: 58.

Eustrophus niger Melsheimer, 1846: 58; Leng, 1920: 238; LeSage, 1991: 246; Poole and Gentili, 1996: 299.

Diagnosis Adults of E. tomentosus are more parallel-sided than other Nearctic Eustrophinae. Individuals also have the following diagnostic features: eyes widely separated; antennal sensilla only partly annular; dorsal pubescence distinctly golden or brown; meso- and metatibiae with ridges; prothoracic episterna without transverse suture. The only other Nearctic species of Eustrophinae with the combination of ridged tibiae and widely separated eyes is Eustrophopsis confinis, which is darker in color and has the transverse suture present on the pro-episterna.

Description TL 4.5-6.0 mm; GEW 2.1-3.0 mm. Body (Fig. 4) oval, parallelsided; dorsal color dark brown, with golden sheen due to dense pubescence; venter and antennae uniformly dark rufous, lighter than dorsal color; dorsal pubescence relatively short, but dense, giving distinct sheen (almost irridescent); eyes (Fig. 11) widely separated (space $-1.5 \mathrm{x}$ length of first antennomere), inner margins deeply emarginate; antennomeres (Fig. 20) 2-11 only slightly but evenly widened to apex, without distinct change in size between any 2 adjacent antennomeres; distal antennomeres subtriangular to nearly quadrate; antennal sensilla not completely annular, present on short sides of antennomeres only (Fig. 24); last maxillary palpomere slightly se- 
curiform; procoxal process triangular, narrowed distally, extended to slightly short of posterior margin of procoxae; prothoracic episternal suture absent; elytral punctation relatively fine, punctures arranged in longitudinal striae; meso- and metatibiae with oblique ridges present (Fig. 15).

Distribution (Fig. 28) The Canadian distribution of this species is fairly broad west to east, although spotty; in the west, specimens are known from British Columbia and southern Manitoba but absent from Alberta and Saskatchewan. In the east, most localities are from southern Ontario and Quebec, with recently documented records from Nova Scotia (Majka and Pollock, 2006). In the United States, the species is distributed primarily east of the Mississippi, but also exhibits the same western gap seen in the Canadian distribution. US distribution: AL, AR, AZ, CA, CT, DC, FL, GA, IA, ID, IL, IN, KS, KY, LA, MD, MI, MN, MO, MS, NC, ND, NH, NJ, NY, OH, OK, OR, PA, SC, TN, TX, VA, VT, WI, WV.

Natural history Label data: Pseudotsuga taxifolia (BC), from fungus (BC), evening flight, 4.IX (BC), ex Populus trichocarpa (BC), in bark Larix occidentalis (BC), fleshy fungus on tree $(\mathrm{ON})$, under bark of pine $(\mathrm{ON})$, under wet moldy bark on dead tree $(\mathrm{ON})$, elm (PQ), UV light trap (PQ), cut surface of stump (PQ), Ulmus americana (PQ), under bark of pine (AL), Spongipellis unicolor (AR), Climacodes septentrionale (AR), Trametes versicolor (AR), under pine bark (CA), Lindgren trap with turpentine bait (CA), on Pinus ponderosa (CA), ex. Polyporus fungus (FL), UV light in oak-maple forest (IA), fungus on dead pine (ID), under bark of old pine stump (MD), in Peromyscus nest debris under bark of dead standing Liriodendron (MD), fungus on oak (MN), funnel traps (MT), at black light, VI.1970 (NC), malaise trap, VIII-ix (NE), window trap, 8-14.VI (NH), light trap, VI (NY), Lindgren funnel trap, alpha-pinene and ethanol lure (OR), stale molasses trap (SC), at wound on oak trunk (SC), malaise in mature hardwood forest, VI (SC), under bark of old dead deciduous tree (VT), ex dead oak stump (WI), under bark of chestnut oak (WV). Chantal (1985) stated that adult E. tomentosus are found under bark of dead trees, particularly elm (Ulmus americana), as well as at sap flows ("exudations"); they are also attracted to light.

Material examined Specimens were examined from the following Canadian localities: BRITISH COLUMBIA: Canal Flats, 21.VIII.1977, B.F. \& J.L. Carr, lot 1, (CARR, 4); same locality, 21.VIII.1977, B.F. \& J.L. Carr, lot 1, (DAPC, 1); Creston, 17.V.1931, G. Stace Smith, (CNC, 5); same locality, 19.V.1956, G. Stace Smith, (CNC, 1); same locality, 17.V.1931, G. Stace Smith, (UBC, 11); same locality, 22.VII.1948, G. Stace Smith, from fungus, (UBC, 1); same locality, 12.VII.1946, G. Stace Smith, host: Pseudotsuga taxifolia, (UBC, 1); same locality, 3.VIII.1945, G. Stace Smith, in fungi, (UBC, 1); same locality, 5.VIII.1950, G. Stace Smith, from fungus, (UBC, 1); same locality, 17.V.1957, G. Stace Smith, evening flight, (UBC, 1); same locality, 4.IX.1951, G. Stace Smith, evening flight, (UBC, 1); same locality, 17.VI.1951, G. Stace Smith, ex fungus, (UBC, 1); same locality, 11.IX.1951, G. Stace Smith, ex fungus, (UBC, 1); same locality, 1.VI.1952, G. Stace Smith, ex fungus, (UBC, 1); same locality, 25.VI.1958, G. Stace Smith, ex fungus, (UBC, 1); same locality, 6.V.1953, G. Stace Smith, evening flight, (UBC, 1); same locality, 18.V.1951, 
G. Stace Smith, ex Populus trichocarpa, (UBC, 1); same locality, 15.VI.1948, G. Stace Smith, from fungus, (UBC, 1); same locality, 22.VII.1948, G. Stace Smith, from fungus, (UBC, 1); Creston, 1900 ft., 25.V.1933, G. Stace Smith, surface of pond, (UBC, 1); Creston, 2000 ft., 20.II.1932, G. Stace Smith, in bark Larix occidentalis, (UBC, 1). Gabriola, 29.IV.1993, B.F. \& J.L. Carr, lot 1, (CARR, 5); same locality, 29.VI.1993, B.F. \& J.L. Carr, (GJHC, 3). MANITOBA: Winnipeg, Univ. of Manitoba campus, forest along Red River, 5-8.VII.1985, D.A. Pollock, suction trap, (DAPC, 1). ONTARIO: no other data, (MCZ, 1). Arnprior, 26.V.1935, W.J. Brown, (CNC, 1); same locality, 9.VI.1935, W.J. Brown, (CNC, 1); Bell's Corners, 17.X.1962, D.D. Munroe, (CNC, 3); same locality, 10.VII.1963, D.D. Munroe, (CNC, 1); Chaffeys Locks Biol. Stn., 6.VI.1986, A. Davies, berlese fleshy fungi on tree, (CNC, 1); Constance Lake, 10.VI.1989, G. Hilchie, ex mushroom, (CARR, 1); DeCew Falls, 6.V.1942, S.D. Hicks, (CNC, 1); Hamilton, 15.VI.1982, M. Sanborne, (CNC, 1); same locality, 28.VI-14.VII.1982, M. Sanborne, (CNC, 2); same locality, 30.VI-8.VII.1981, M. Sanborne, (CNC, 1); same locality, 23.VII.1980, M. Sanborne, Malaise trap, (CNC, 1); Lambton Co., Pinery Prov. Park, 15-20.VI.1994, J. Skevington, Malaise Cedar trail oak savanna meadow, (DEBU, 2); same locality, 22.VI.1995, G. Vogg, Visitor Centre, (DEBU, 1); Lanark, 17.IX.1977, S.A. Marshall, (JBWM, 3); Leamington, 18.VI.1940, W.J. Brown, (CNC, 1); Merivale, 4.VI.1935, W.J. Brown, (CNC, 1); Ottawa, 14.VIII.1912, Beaulieu, (CNC, 2); same locality, (CNC, 4); same locality, 17.V, under bk. of pine, (CNC, 3); same locality, 14.VIII.1912, Beaulieu, (MCZ, 2); Prince Edward Co., 15.VI.1947, Brimley, (CARR, 3); same locality, 23.IV.1922, Brimley, (CNC, 1); same locality, 21.VII.1918, Brimley, (CNC, 1); same locality,

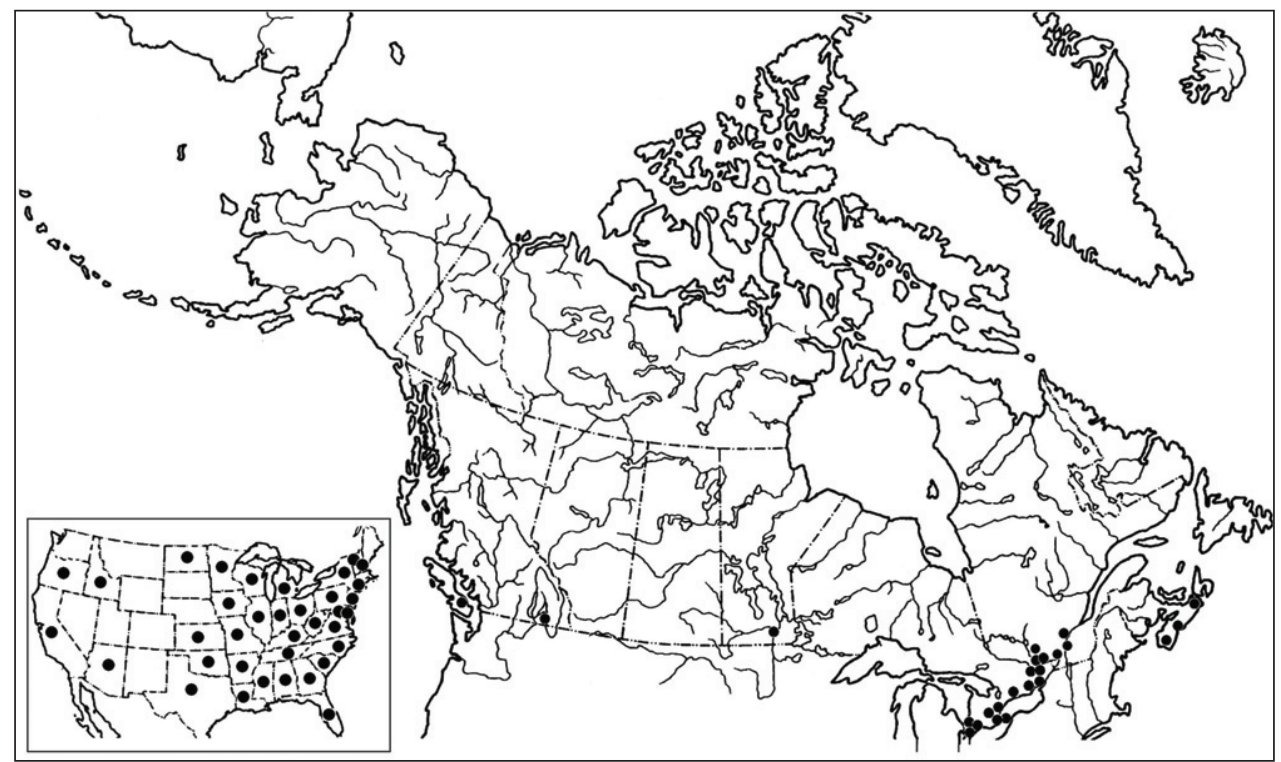

Fig. 28. Canadian distribution of Eustrophus tomentosus. Inset shows US distribution, by state. 
20.VII.1938, Brimley, (CNC, 1); same locality, 13.VI.1940, Brimley, (CNC, 1); same locality, 16.IX.1923, Brimley, (CNC, 1); same locality, 21.VII.1918, Brimley, (CNC, 1); same locality, Brimley, (UAIC, 3); same locality, Brimley, (UAIC, 3). Rondeau Pk., 2-6.VI.1985, L. LeSage \& A. Smetana, flight intercept in white pine stand, (CNC, 3); Rondeau Pk., 2-6.VI.1985, L. LeSage \& A. Smetana, int. trap in maple-beech forest, (CNC, 1); same locality, 14.VI-2.VII.1985, L. LeSage \& D.M. Wood, (CNC, 1); Rondeau Pr. Pk., Black Oak Trail, 5.VI.1985, A. Davies \& J.M. Campbell, under wet moldy bk. on dead tree, (CNC, 1); Toronto, R.J. Crew, (CUIC, 2); same locality, 2.I.1895, R.J. Crew, (CUIC, 4); same locality, III, Crew, (UWEM, 1); Trenton, 25.V.1899, Evans, (CNC, 1); no other data, (DEBU, 2); Ariss, 18.VII.1976, S.A. Marshall, (DEBU, 1); Gananoque, 22.VI.1963, C.J. Edwards, (DEBU, 1); Grimsby, 14.VI.1977, W.A. Attwater, (DEBU, 2); Guelph, 17.VII.1976, S.A. Marshall, (DEBU, 5); same locality, 27.III.1977, Kevin Barber, (DEBU, 10); Lanark, 2.VIII.1976, M.J. Sharkey, (DEBU, 1); London, W. Saunders, (DEBU, 3); same locality, 28.VI.1976, W.A. Attwater, (DEBU, 1); Oakville, 21.VII.1977, W.A. Attwater, (DEBU, 1); Port Credit, 16.V.1993, C. Krupke, rotting wood, (DEBU, 1); Pr. Edw. Co., Brimley, 2.VI.1940, (AMNH, 1); Primrose, 27.VII.1977, W.A. Attwater, (DEBU, 1); Ridgeway, (DEBU, 1); Whitby, 21.IV.1974, G.J. Umphrey, (DEBU, 1). QUEBEC: Aylmer, 12.V.1932, W.J. Brown, (CNC, 1); Gatineau Pk., 24.V.1970, J.M. Campbell, (CNC, 1); Montreal, 4.IV.1969, E.J. Kiteley, elm, (CNC, 1); same locality, 27.VIII.1973, E.J. Kiteley, UV light trap, (CNC, 1); same locality, 21.VII.1974, E.J. Kiteley, UV light trap, (CNC, 1); same locality, 25.VI.1980, E.J. Kiteley, cut surface stump, (CNC, 1); same locality, 26.VIII.1973, E.J. Kiteley, UV light trap, (CNC, 1); Old Chelsea, NCC woodpile, 27.V-5.VI.1987, L. Masner, pans, (CNC, 1); Riviera Touraine, 12.IV.1974, R. Sexton, Ulmus americana, (CNC, 15); same locality, 14.IV.1974, R. Sexton, Ulmus americana, (CNC, 19); Terrebonne, Saint-Hippolyte, 27.V.1978, S. Laplante, UV light, (SLC, 1). LOCALITY UNKNOWN. no other data, (CUIC, 1); Ulke collection, (CMNH, 1); no other data, (USNM, 1). NOVA SCOTIA: Guysborough Co., Borneo, 28.VI.1995, C. Corkum, (NSMC, 1); Halifax Co., Big St. Margarets Bay, 29.VII-13.VIII.1997, D.J. Bishop, in old red spruce habitat, (NSMC, 1); Queens Co., Tobeatic Lake, 3.VI.2003, P. Dollin, red spruce habitat (80-120 yrs.), (NSMC, 2)

Eustrophopsis Champion, 1889

Eustrophopsis Champion, 1889: 77.-Type species: Orchesia quindecimmaculatus Laporte, 1840; Champion, 1915: 138; Champion, 1916: 1, 138; Csiki, 1924: 7; Blackwelder, 1945: 494; Nikitsky, 1998: 58; Young and Pollock, 2002: 416.

Eustrophinus Seidlitz, 1898: 438, 440.-Type species: Mycetophagus bicolor Fabricius, 1798; Champion, 1916: 3; Leng, 1920: 238; Leng and Mutchler, 1933: 36; Csiki, 1924: 8; Blackwelder, 1945: 495; Hatch, 1965: 66; Poole and Gentili, 1996: 299; LeSage, 1991: 246; Nikitsky, 1998: 58; Young and Pollock, 2002: 416.

Eustrophus Champion, 1889: 75, nec. Illiger, 1802. 
Champion (1889) erected Eustrophopsis to include species of "Eustrophi" that exhibited a deeply emarginate prosternal process to receive a sharply keeled mesosternum. Seidlitz (1898) included in Eustrophinus, species that lacked this emarginate prosternal process and keeled mesosternum. However, according to Nikitsky (1998), who has studied this group extensively, these characters exhibit a graded transition without a distinct morphological gap. Therefore, he sunk the name Eustrophinus as a junior synonym of Eustrophopsis Champion.

Eustrophopsis is the most diverse genus of Eustrophinae, with approximately 55 species known from Afrotropical, Neotropical and Nearctic regions. Nikitsky (1998: 58) stated that it is "remarkable that Eustrophopsis seems to be absent both from the Oriental Region and Palearctic. It may be so that it is replaced there by species of the genera $\mathrm{Ho}$ lostrophus and Synstrophus unknown from the Neotropical and Afrotropical regions".

\section{Eustrophopsis bicolor (Fabricius, 1798)}

(Figs 5, 12, 21, 29)

Mycetophagus bicolor Fabricius, 1798: 497 (type(s) not examined); Melsheimer, 1853: 143; LeConte, 1873: 335; Provancher, 1877: 467; Horn, 1888: 35; Weiss, 1919: 133; Leng, 1920: 238; Csiki, 1924: 8; Viedma 1971; Poole and Gentili 1996: 299; LeSage 1991: 246. According to LeConte (1873: 335) "the proper authority for this species is Say, its first describer; Mycetophagus bicolor Fabr. is probably a Platydema". I have seen no other such reference, and all sources consulted have listed Fabricius as the author of this species; this was done even by Say (1826).

Diagnosis Members of this species are readily distinguished by the following combination of features: outer surface of meso- and metatibiae with numerous transverse ridges; eyes narrowly separated; much of ventral surface reddish, contrasting darker dorsal color; antennomere 11 yellow-red, exhibiting a distinct contrast to antennomeres 5-10.

Description TL 4.2-6.5 mm; GEW 2.0-3.2 mm. Body (Fig. 5) elongate oval, moderately tapered posteriorly; dorsal color dark piceous to black; antennae tricolored: antennomeres 1-4 red, 5-10 piceous, antennomere 11 yellow-red, distinctly contrasting against preceding darker antennomeres; maxillary palpi similar in color to basal antennomeres; venter a combination of dark (same as dorsum) and lighter colored sclerites (abdominal ventrites in most specimens contrasting darker color of thorax); dorsal pubescence relatively long, conspicuous; eyes narrowly separated (Fig. 12), or almost contiguous (space < length of antennomere 1), inner margin moderately emarginate; antennomeres (Fig. 21) 2-4 short, submoniliform, antennomeres 5-10 widened, becoming more triangular toward antennomeres 9-10; antennal sensilla completely annular (as in Fig. 25); last maxillary palpomere not modified; procoxal process triangular, narrowed distally, extended to slightly short of posterior margin of procoxae; prothoracic episternal suture present; elytral punctation coarse, punctures arranged in longitudinal striae (Fig. 5); meso- and metatibiae with oblique ridges present (as in Fig. 15). 
Distribution (Fig. 29) This is one of the most widely distributed Nearctic species of Eustrophinae, with a primarily eastern distribution. In Canada, it has not been collected west of Winnipeg, MB and extends east to Quebec. In the United States, it seems to have a mainly eastern distribution, although specimens have been collected in Arizona, California and Idaho. Specimens are also known from Mexico. US distribution: AL, AR, AZ, CA, CO, CT, DC, DE, FL, GA, IA, ID, IL, IN, KS, KY, LA, MA, MD, MI, MN, MO, MS, NC, ND, NE, NJ, NM, NY, OH, OK, PA, RI, SC, TN, TX, VA, VT, WI, WV, WY.

Natural history Chantal (1985: 53) noted the species as occurring under dead tree bark and also gave the following as fungal substrates on which adults were collected: Pleurotus sapidus, Polyporus squamosus, $P$. betulinus, $P$. versicolor, $P$. confragosa. Adults of E. bicolor are known to be often found in the same micro-habitats as $S$. repandus (Chantal 1985; Pollock, pers. observ.). In the summer of 2005, the author found multiple adults of E. bicolor and S. repandus on and in a rotting fungusy log near Lockport, MB. From 2006 to 2008, specimens were collected on the same log; in the summer of 2008, E. bicolor was absent, but S. repandus were still present. Label data: underside of fungusy, dead log at night (MB), on piece of cut wood at night (MB), fungusy stump of Acer negundo (MB), bracket fungus (ON), in rotting Pleurotus (PQ), on trunk of dead Ulmus americana (PQ), under bark of dead maple (PQ), under bark of rotting trunk of Pinus eliottii with polypore fungi (Bahamas), ex Fomes on Salix (Bahamas), under bark of Fagus (AR), Trametes versicolor (AR), Meripilus giganteus (AR), polypore tree fungus (AR), under rotting oak bark (CT), in litter at base of dying Ailanthus (CT), large orange polypore shelf fungus [? Polyporus sulphureus] on standing tree trunk (CT), Omphalotus olearius (CT), under bark (DE), shelf fungi (FL), Griffolia fungus (FL), Polyporus hypnoides (FL), Polyporus sulphureus (FL, MA), under bark of dead pine (FL), hibernating under bark (GA), under bands of tar paper on apple trees (IL), Armillariella mellea (GA), Pleurotus ostreatus (IL), on shelf fungi (KS), in sweetgum stump (LA), Polyporus adustus (LA), Panus rudis (MA, NJ), under bark of dead Pinus virginiana (MA), under bark dead standing Quercus (MA), in Peromyscus nest debris under bark dead standing Liriodendron (MA), under bark of stump of Prunus serotina (MA), fungus on bark (MS), injured cypress (NC), oak (NC), under bark dead standing pine (NC), under oak bark (NC), Pleurotus sp. (NC, OK), in mushroom (NC), Trametes hispida (ND), under hardwood bark (NJ), ex fungus on Mimosa stump (NJ), on polypore on dead Quercus (TX), polypore fungus (UT), Polyporus squamosus (VT), under poplar bark (WI).

Material examined Specimens were examined from the following Canadian localities: MANITOBA: Winnipeg, King's Park, D.A. Pollock, sitting on underside of fungusy, dead log at night, (DAPC, 1); same locality, 13.VIII.1998, D.A. Pollock, sitting on piece of cut wood at night, (DAPC, 1); Winnipeg, Univ. of Manitoba campus, nr. apiary, $49^{\circ} 48^{\prime} 30^{\prime \prime} \mathrm{N}, 97^{\circ} 07^{\prime} 35^{\prime \prime} \mathrm{W}, 27 . \mathrm{VII} .1999$, D.A. Pollock, on thin white fungus on underside of small log, (DAPC, 7); Winnipeg, University of Manitoba campus, along Red River, 25-28.VIII.1996, D.A. Pollock, at night, on fungusy stump of Acer negundo, (DAPC, 1); Winnipeg, University of Manitoba campus, forest along Red River (south), 11.VII.1999, D.A. Pollock, crawling on fungusy stump of Acer negun- 
do at night, (DAPC, 1). ONTARIO: Bolton, 4.V.1978, R.S. Anderson, (ROME, 1); Chatham, 23.VIII.1929, G.M. Stirrett, (CNC, 1); Dunn Twp, 29.VIII.1971, W.W. Judd, (CNC, 1); Dunnville, 24.VIII.1938, S.D. Hicks, very teneral, (CNC, 2); Grand Bend, 6.VII.1939, T.N. Freeman, (CNC, 1); Guelph, 23.VIII.1990, Heather Dewar, bracket fungus, (DEBU, 4); Lanark, 2.VIII.1979, S.D. Hicks, (CNC, 1); same locality, 4.VII.1976, S.D. Hicks, (CNC, 1); Leamington, 18.VI.1940, W.J. Brown, (CNC, 1); same locality, 24.V.1970, T.D. Galloway, (JBWM, 1); Ottawa, 4.IX.1912, Beaulieu, 1 teneral, (CNC, 3); same locality, 2.X.1912, Beaulieu, (CNC, 1); same locality, 14.VIII.1912, Beaulieu, (CNC, 1); same locality, 9.IX.1912, Beaulieu, (MCZ, 3); Peel Co., Port Credit, 6-11.VIII.1965, I.M. Smith, (ROME, 1); Pelee Is., 3.VII.1931, W.J. Brown, (CNC, 1); Pelee Island, 1.VII.1940, W.J. Brown, slightly teneral, (CNC, 1); Pelee Island, Lake Erie, 17.VII.1981, S.W. Nichols, (CUIC, 4); Pelee Island, marsh on SE shore, 14.VIII.1980, S.W. Nichols, flooding shore of sand spit, (CUIC, 1); Point Pelee, 26.VII-4.VIII.1999, A. Tesolin, malaise \& pan trap $41^{\circ} 59^{\prime} \mathrm{N}, 82^{\circ} 27.5^{\prime} \mathrm{W}$, (DEBU, 2); Prince Edward Co., 21.V.1916, Brimley, (CNC, 1); same locality, 3.VI.1917, Brimley, (CNC, 1); same locality, 31.VII.1938, Brimley, (CNC, 1); same locality, 23.IV.1922, Brimley, (CNC, 1); same locality, 21.V.1916, Brimley, (CNC, 1); same locality, 20.V.1923, Brimley, (CNC, 1); same locality, 7.VII.1946, Brimley, (CNC, 1); same locality, 3.VI.1923, Brimley, (CNC, 1); same locality, 18.IX.1921, Brimley, (CNC, 3); Rondeau Prov. Pk., 2.VIII.1982, L. LeSage, ex. Polyporus, (CNC, 9); Toronto, R.J. Crew, (CUIC, 2); same locality, 24.VIII.1895, R.J. Crew, (CUIC, 1); same locality, 12.IX.1908, R.J. Crew, (ROME, 1); Wheatley, 22.VII.1965, (FSCA, 2); Windsor, 2-16.VIII.1982, S. Marshall, (CNC, 1); Essex Co., Point Pelee Natl. Pk. Forested area by W beach, 21.VIII.1999, O. Lonsdale, malaise/pan traps, (DEBU, 1); Pt. Pelee, 18.VII.1978, J.M.

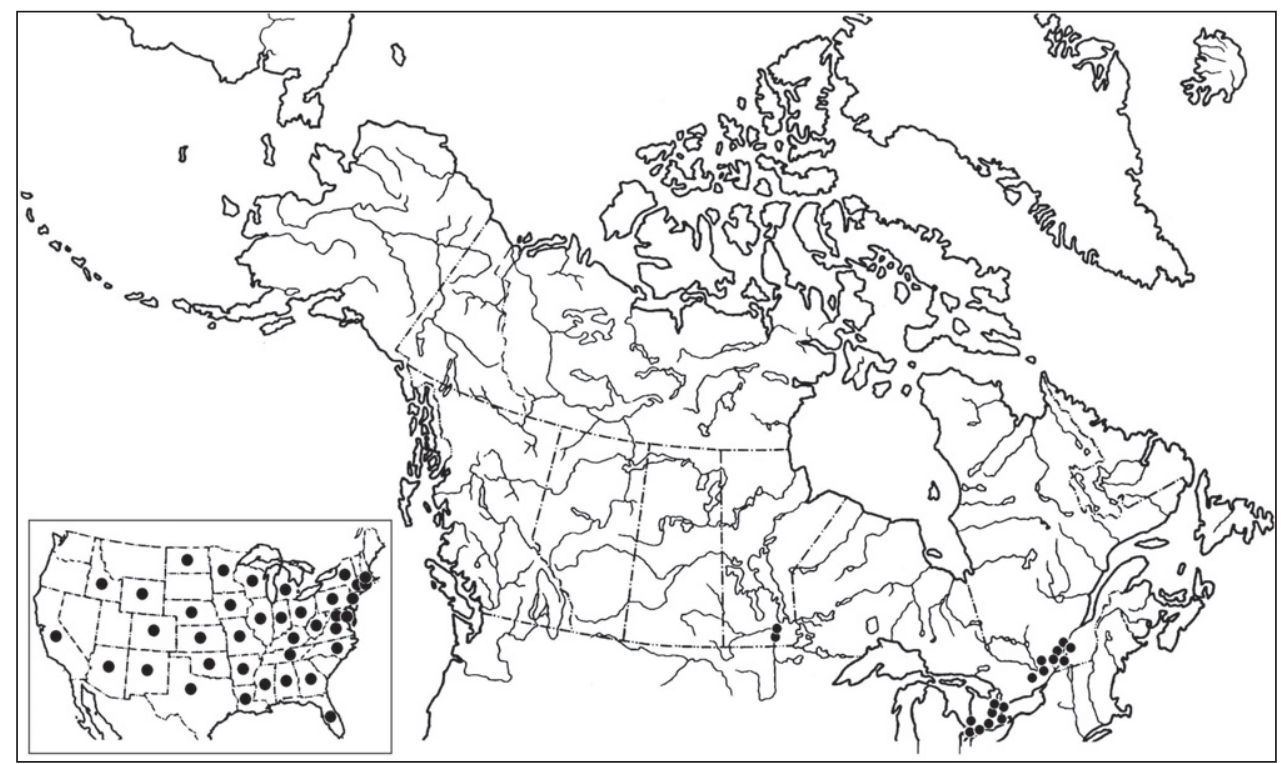

Fig. 29. Canadian distribution of Eustrophopsis bicolor. Inset shows US distribution, by state. 
Cumming, (DEBU, 1); Dundas, 15.VII.1975, J.M. Cumming, (DEBU, 1); Guelph, 25.VII.1977, A.A. Konecny, (DEBU, 3); same locality, 3.VIII.1976, Paul R. Heels, (DEBU, 1); same locality, 25.IV.1973, R.E. Roughley, (DEBU, 1); London, 19.V.1975, J.M. Heraty, (DEBU, 1); Pr. Edw. Co., Brimley, 2.VI.1940, (AMNH, 1); same locality, 31.VII.1938, (AMNH, 1); Pt. Pelee Nat. Pk., 13.VIII.1983, S. Marshall, C. Logan, \& S. Girigsby, (DEBU, 1); Tilbury, 30.IX.1965, Rosenberg collection, (USNM, 1); Toronto, 19.VII.1976, W.A. Attwater, (DEBU, 1); same locality, 10.VII.1977, W.A. Attwater, (DEBU, 1); same locality, 15.VIII.1981, Lonny Coote, (DEBU, 1). QUEBEC: Aylmer, 25.V.1934, W.J. Brown, (CNC, 1); Chambly, Longueuil, 14.VIII.1976, S. Laplante, in rotting Pleurote, (SLC, 2); Deux-Montagnes, Parc Paul-Sauvé, 29.IV.1990, S. Laplante, (SLC, 1); Gatineau Pk. nr. Meach L., 1.IX.1982, A. Davies, berlese fleshy fungi on tree, (CNC, 1); Gatineau, Aylmer, 28.V.1991, S. Laplante, on trunk of dead Ulmus americana at 22:00, (SLC, 2); Huntingdon, Havelock, 14.V.1981, S. Laplante, (SLC, 1); Lacolle, 10.VIII.1945, W.J. Brown, (CNC, 4); Missisquoi, Scottsmore, 9.VI.1979, S. Laplante, (SLC, 1); Montreal, 5.VIII.1985, E.J. Kiteley, UV light trap, (CNC, 1); same locality, 16.VII.1967, E.J. Kiteley, (CNC, 1); same locality, 13.VI.1984, E.J. Kiteley, UV light trap, (CNC, 2); same locality, 2.IX.1969, E.J. Kiteley, (CNC, 1); same locality, 26.VI.1967, E.J. Kiteley, (CNC, 1); same locality, 28.V.1967, E.J. Kiteley, (CNC, 1); same locality, 17.IX.1972, E.J. Kiteley, (CNC, 1); same locality, 3.VII.1970, E.J. Kiteley, under bark, (CNC, 1); same locality, 6.VII.1976, E.J. Kiteley, UV light trap, (CNC, 1); same locality, 23.VI.1968, E.J. Kiteley, on log, (CNC, 1); same locality, 28.VIII.1972, E.J. Kiteley, UV light trap, (CNC, 1); same locality, 8.VII.1978, E.J. Kiteley, UV light trap, (CNC, 1); same locality, 28.VII.1980, E.J. Kiteley, UV light trap, (CNC, 1); same locality, 17.VII.1977, E.J. Kiteley, UV light trap, (CNC, 1); Rigaud For. at Ottawa River, 11.V.1988, A. \& Z. Smetana, (CNC, 2) Vaudreuil, Rigaud, 21.V.1982, P. Bélanger, under bark of dead maple, (CMNC, 1).

\section{Eustrophopsis confinis (LeConte, 1866)}

(Figs 6, 13, 22, 30)

Eustrophus confinis LeConte, 1866: 152.—Horn, 1888: 35; Dury, 1906: 260; Leng, 1920: 238; Csiki, 1924: 10; Hatch, 1965: 66; Poole and Gentili, 1996: 299; LeSage, 1991: 246; Majka and Pollock, 2006: 53. Lectotype (here designated), sex unknown, labelled "Wis / Type 4780 / E. confinis Lec", in MCZ.

Diagnosis Eustrophopsis confinis is the only Nearctic species of Eustrophinae with a combination of widely separated eyes, and transverse ridges on the outer surface of the meso- and metatibiae. In fact, it may be the only world Eustrophopsis with widely separated eyes. Also, its almost uniformly dark color separates E. confinis from E. bicolor.

Description TL 4.8-6.1 mm; GEW 2.4-3.1 mm. Body (Fig. 6) ovate, subparallelsided; dorsal and ventral color uniformly dark piceous to black; antennomeres 1-4 and 11 slightly lighter in color than antennomeres 5-10; dorsal pubescence relatively long, 
conspicuous; eyes (Fig. 13) widely separated (space 1-1.5 x length of antennomere 1), inner margin deeply emarginate; antennomeres (Fig. 22) 5-10 subquadrate; antennal sensilla completely annular (as in Fig. 25); last maxillary palpomere unmodified, fusiform; prosternal process triangular, moderately narrowed distally, extended to slightly short of posterior margin of procoxae; prothoracic episternal suture present; elytral punctation coarse, punctures arranged in longitudinal striae (Fig. 6); meso- and metatibiae with oblique ridges (as in Fig. 16).

Distribution (Fig. 30) Although specimens are seemingly rarely collected, E. confinis exhibits one of the broadest west-east ranges. The species is known from western British Columbia to Nova Scotia. The single record from Saskatchewan is the most northerly known specimen of Eustrophinae in North America. US distribution: ID, MI, ME, NE, NH, SD, WI.

Natural history Label data: Polyporus anceps (ME). Chantal (1985) stated that no natural history data were known to him, but that E. confinis should be similar to other members of the group.

Material examined Specimens were examined from the following Canadian localities: BRITISH COLUMBIA: McIntyre Cr., Oliver, 6.VI.1959, R.E. Leech, (CNC, 1); Radium, 4.VI.1953, B.F. \& J.L. Carr, lot 5, (CARR, 3); same locality, 4.VI.1953, B.F. \& J.L. Carr, lot 5, (DAPC, 2); Trinity Valley, 16.VIII.1927, J.R.L. Howell, (PFC, 1); Vernon, 11.V.1943, H. Leech, on grass, (UBC, 1); Victoria, (MCZ, 1); Tappen, 3.VI.1978, B.F. \& J.L. Carr, lot 1, (DAPC, 1). SASKATCHEWAN: Road 914, $82 \mathrm{~km}$ N. Jct. Hwy. 165, 30.VII.1986, B.F. \& J.L. Carr, lot 2, (DAPC, 1). MANITOBA: Duck Mtn. Prov. Pk., 19-24.VI.1981, Ashworth, Schwert \& Keller, open gravelly mud with sparse vegetation by water's edge, (DAPC, 1); Riding Mt. Pk., 2.VI.1938, W.J.

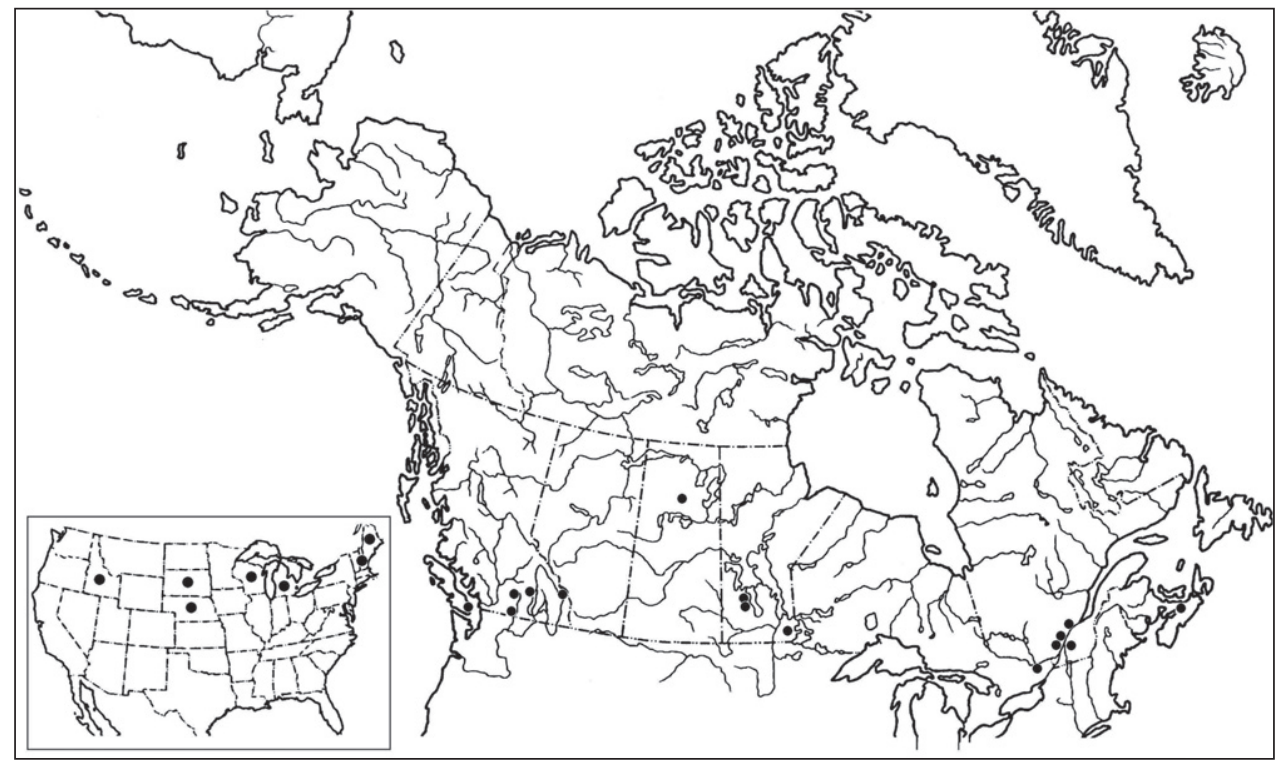

Fig. 30. Canadian distribution of Eustrophopsis confinis. Inset shows US distribution, by state. 
Brown, (CNC, 1); Sandilands Forest, 15.VIII.1993, N.J. Holliday, (JBWM, 1). ONTARIO: Constance Bay, 16.V.1933, W.J. Brown, (CNC, 1). NOVA SCOTIA: Cumberland Co., Wentworth, 21.V-5.VII.1965, B. Wright, (NSMC, 2).

\section{Synstrophus Seidlitz, 1898}

Synstrophus Seidlitz, 1898: 438.-Type species: Eustrophus macrophthalmus Reitter, 1887 (orig. des.); Hatch 1965: 66; LeSage 1991: 246; Poole and Gentili 1996: 300; Nikitsky, 1998: 58; Young and Pollock, 2002: 416.

There are five species of Synstrophus (Nikitsky, 1998) distributed in China, Japan, Oriental region, and North America. Nikitsky (1998) mentioned that, based on several characters, $S$. repandus (Horn) might eventually merit placement in a different genus-group taxon.

Synstrophus repandus (Horn, 1888)

(Figs 7, 14, 23, 25, 31)

Eustrophus repandus Horn, 1888: 33.—Csiki, 1924: 9; Hatch, 1965: 66, Plate VIII, fig. 9; LeSage, 1991: 246; Poole and Gentili, 1996: 300; Young and Pollock, 2002: 416. Lectotype, sex unknown, labelled "Pen / LectoTYPE / Eustrophus repandus Horn / MCZ Type 34039”, in MCZ. Paralectotypes (3), in MCZ.

Diagnosis The smooth meso- and metatibiae (no oblique ridges), overall dark body color, and narrowly separated eyes serve to separate this species from all other Nearctic Eustrophinae.

Description TL 5.6-7.0 mm; GEW 2.5-3.4 mm. Body (Fig. 7) ovate, only somewhat tapered posteriorly; dorsal color dark piceous to black; antennae with basal 4 antennomeres dark rufous, distal half of antennomere 11 rufous; venter at least slightly lighter in color than dorsum; mouthparts similar in color to antennomeres 1-4; legs and abdominal ventrites dark rufous to piceous; dorsal pubescence relatively long, conspicuous; eyes (Fig. 14) narrowly separated, or almost contiguous (space < length of antennomere 1), inner margin moderately emarginate; antennomeres (Fig. 23) 2-4 short, submoniliform; antennomeres 5-10 widened, becoming more triangular toward antennomeres 9-10; antennal sensilla completely annular (Fig. 25); last maxillary palpomere unmodified, fusiform; procoxal process triangular, narrowed distally, extended to slightly short of posterior margin of procoxae; prothoracic episternal suture absent; elytral punctation coarse, punctures arranged in longitudinal striae (Fig. 7); meso- and metatibiae with scattered short spines, but distinct ridges absent (as in Fig. 15).

Distribution (Fig. 27) The distribution of this species is very similar to that of $E$. tomentosus, in that most specimens are known from British Columbia and southern Ontario and Quebec, with a few localities in Manitoba. No specimens of this species have 
been seen from Alberta or Saskatchewan. US distribution: AL, AR, CA, CT, DC, DE, FL, GA, IA, ID, IL, IN, KS, KY, LA, MA, MD, ME, MI, MN, MO, MS, MT, NC, ND, NE, NH, NJ, NY, OH, OK, OR, PA, SC, SD, TX, UT, VA, WA, WI, WV, WY.

Natural history Label data: Pinus ponderosa bark (BC), shelf fungus on birch (BC), fungus on cottonwood (BC), Populus trichocarpa (BC), ex fungus on Betula (BC), underside of Populus trichocarpa log $(\mathrm{BC})$, fleshy bracket fungus $(\mathrm{ON})$, under wet moldy bark on dead tree (ON), Pleurotus ostreatus (IL, MA, PQ), polypore on branch of old Quercus rubrus (PQ), under dead pine bark (AL), Meripilus giganteus (AR), ex fungi and under bark (FL), large mushroom on log (IL), Polyporus adjustus (IL), Laetiporus sulphureus (IL), under Pinus bark (LA), ex bracket fungi (NC), in slimey fungus under pine bark (NY), under dead bark (SC), ex. Polyporus schweinitzii (VT), under bark of pine (WY).

Material examined Specimens were seen from the following Canadian localities: BRITISH COLUMBIA: $5 \mathrm{~km}$ SW Radium, 24.VIII.1987, B.F. \& J.L. Carr, lot 1, (CARR, 2); same locality, 24.VIII.1987, B.F. \& J.L. Carr, lot 1, (DAPC, 3); Radium, 24.VII.1987, B.F. \& J.L. Carr, (GJHC, 5); 7 mi E. Terrace, 26-27.VI.1968, Campbell \& Smetana, ex river debris, (CNC, 1); Copper Mountain, 21.VIII.1927, G. Stace Smith, (JBWM, 3); Copper Mtn., 21.VIII.1927, G. Stace Smith, Pinus ponderosa bark, (UBC, 4); Creston, 11.V.1958, H. \& A. Howden, shelf fungus on birch; $1 \mathrm{spec}$. slightly teneral, (CNC, 8); same locality, 14.V.1958, H. \& A. Howden, fungus on cottonwood, (CNC, 1); same locality, 6.IX.1951, G. Stace Smith, ex fungus, (UBC, 1); same locality, 16.VIII.1951, G. Stace Smith, ex fungus, (UBC, 1); same locality, 7.IX.1951, G. Stace Smith, ex fungus, (UBC, 1); same locality, 25.X.1951, G. Stace Smith, under log, (UBC, 1); same locality, 21.V.1951, G. Stace Smith, Populus trichocarpa, (UBC, 1); same locality, 12.X.1951, G. Stace Smith, ex fungus, (UBC, 1); same locality, 17.V.1951, G. Stace Smith, ex fungus, (UBC, 1); same locality, 20.X.1951, G. Stace Smith, logs, (UBC, 1); same locality, 23.V.1951, G. Stace Smith, ex fungus on Betula, (UBC, 1); same locality, 26.V.1951, G. Stace Smith, ex fungus on Betula, (UBC, 2); same locality, 9.IX.1951, G. Stace Smith, ex fungus, (UBC, 1); same locality, 22.VII.1933, G. Stace Smith, underside of Populus trichocarpa log, (UBC, 1); same locality, 12.X.1946, G. Stace Smith, in fungus, (UBC, 1); same locality, 8.VI.1950, G. Stace Smith, ex fungus Populus trichocarpa, (UBC, 1); same locality, 23.VII.1948, G. Stace Smith, from fungus, (UBC, 1); same locality, 18.V.1948, G. Stace Smith, from fungus, (UBC, 2); same locality, 7.XII.1931, G. Stace Smith, on cordwood, (UBC, 1); same locality, 22.VII.1948, G. Stace Smith, from fungus, (UBC, 2); Creston, 2200 ft., 18.III.1933, G. Stace Smith, decaying wood of Pinus ponderosa, (UBC, 1); Gabriola, 2.VI.1994, B.F. \& J.L. Carr, lot 7, (CARR, 1; GJHC, 1); same locality, 29.IV.1993, B.F. \& J.L. Carr, lot 1, (CARR, 1); same locality, 29.V.1994, B.F. \& J.L. Carr, lot 8, (CARR, 3); same locality, 10.IX.1992, B.F. \& J.L. Carr, lot 2, (CARR, 1); Haney, 2.VII.1962, B.F. \& J.L. Carr, lot 1, (CARR, 1); Lockhart Beach, Kootenay Lk., 12.VI.1962, C.W. O’Brien, (MCZ, 1); Midday Val. Merritt, 21.VI.1926, W. Mathers, (CNC, 1); same locality, 20.IV.1926, R. Hopping, old stumps of P. ponderosa, (CNC, 1); same locality, 12.VII.1925, K.F. Auden, (CNC, 2); Midday Valley, Merritt, 4-7.VI.1924, K.F. Auden, (INHS, 4); same locality, 7.VI.1924, K.F. Auden, (MCZ, 1); same locality, 4.VI.1924, K.F. Auden, (PFC, 1); same locality, 11.VII.1925, K.F. Au- 
den, (PFC, 1); same locality, 3.IX.1925, W. Mathers, Pinus ponderosa, (PFC, 1); same locality, 10.VI.1926, H.H. Thomas, (PFC, 1); Mirror Lake, 10.VI.1984, B.F. \& J.L. Carr, lot 2, (CARR, 1); Salmon Arm, 27.III.1929, H.B. Leech, (UBC, 1); same locality, v.1934, D.H. Leech, (UBC, 1); same locality, 22.V.1932, H. Leech, fungus, (UBC, 1); Sanca, 8.VIII.1933, G. Stace Smith, on stone wall, (UBC, 1); Sleeping Beauty Mtn., NE Terrace, 1800 m., 1.VII.1987, D. \& K. Larson, (MUNC, 1); Tappen, 3.VI.1978, B.F. \& J.L. Carr, lot 1, (CARR, 9; GJHC, 3); same locality, 3.VI.1978, B.F. \& J.L. Carr, lot 1, (DAPC, 1); Vancouver, 15.III.1896, (CMNH, 5); Vancouver Island, 15.III.1899, (UMRM, 1); Victoria, (INHS, 2); Wynndel, 13.V.1958, H. \& A. Howden, (CNC, 1). MANITOBA: Aubigny, 24.VIII.1987, J.E. Blatta, (JBWM, 2); Aweme, 20.VI.1910, E. Criddle, (JBWM, 1); Sandilands Res, 5 J 1938, D.N. Smith, (CNC, 1); Winnipeg, University of Manitoba campus, honey house, 10-17.VI.1991, R.E. Roughley, suction trap, (JBWM, 1). ONTARIO: no other data, (MCZ, 1); Bell's Corners, 17.X.1962, D.D. Munroe, (CNC, 1); Brant Co., Pinehurst Cons. Area, Hwy 24A, n. of Paris, 21.V.1971, H. Frania, in fleshy bracket fungus in woods, (ROME, 5); Chalk River, 18.VII.1985, J.V.R. Chenier, (CMNC, 1); Constance Bay, 4.IX.1965, D.D. Munroe, (CNC, 1); Dunn Twp, 1.IX.1971, W.W. Judd, (CNC, 1); Essex Co., Point Pelee Natl. Pk. East beach, 8.VI.2000, E. Reichert, (DEBU, 1); same locality, [8.VI.2000], O. Lonsdale, under bark, (DEBU, 1); Essex Co., Windsor, -1.5km S Ojibway Prairie , 1-19.VIII.2001, P. Pratt, prairie remnant, forest edge, malaise trap, (DEBU, 1); Fitzroy Hbr, 31.V.1965, D.D. Munroe, (CNC, 1); Grand Bend, 22.VI.1936, A.A. Wood, (CNC, 1); Hamilton, 3-17. VI.1982, M. Sanborne, (CNC, 1); same locality, 31.VII.1980, M. Sanborne, (CNC, 1); same locality, 7.VI.1981, M. Sanborne, (CNC, 4); same locality, 28.VIII.1980, M. Sanborne, (CNC, 3); same locality, 14-19.VII.1981, M. Sanborne, (CNC, 1); same locality, 14-21.VI.1981, M. Sanborne, (CNC, 1); same locality, 9-19.IX.1981, M. Sanborne, (CNC, 1); same locality, 16.VII.1980, M. Sanborne, (CNC, 1); same locality, 28.VI-14. VII.1982, M. Sanborne, (CNC, 1); same locality, 23.VII.1980, M. Sanborne, Malaise trap, (CNC, 1); same locality, 27.VII-9.VIII.1982, M. Sanborne, (CNC, 2); same locality, 23.V-3.VI.1982, M. Sanborne, (CNC, 1); same locality, 14-26.VII.1982, M. Sanborne, (CNC, 1); Lambton Co., Pinery Prov. Park , 20.VI.1994, J. Skevington, malaise Cedar trail oak savanna meadow, (DEBU, 1); same locality, 26.VI.1994, J. Skevington, malaise Cedar trail oak savanna meadow, (DEBU, 1); Ottawa, (CNC, 4); same locality, 4.VIII, fungus, (CNC, 1); same locality, 22.VIII.1912, Beaulieu, (CNC, 1); same locality, 27.VIII.1912, Beaulieu, (MCZ, 4); Peel Co., Bolton, 4.V.1978, R.S. Anderson, under bark, (ROME, 1); Peel Co., Port Credit, 29.IV.1966, I.M. Smith, (ROME, 3); same locality, 18.VII.1965, I.M. Smith, (ROME, 1); same locality, 24.VII.1965, I.M. Smith, (ROME, 1); same locality, 16.IV.1966, I.M. Smith, (ROME, 2); Prince Edward Co., 30.IV.1922, Brimley, (CNC, 1); same locality, 2.VI.1940, Brimley, (CNC, 2) same locality, 1.VI.1930, Brimley, (CNC, 1); same locality, 31.V.1942, Brimley, (CNC, 1); same locality, 12.X.1919, Brimley, (CNC, 1); same locality, 29.VI.1941, J.F. Brimley, (FMNH, 1); Rainy River Dist., 22.VI.1924, J.F. Brimley, (CNC, 1); Reg.Mun. of Ottawa-Carleston Haldiman, 9.VI.1995, Brendon Larson, Twp. 5 km NW Centreton conch on dead Pop. Trem., (DEBU, 1); Rondeau Pr. Pk., Black Oak Trail, 5.VI.1985, A. Davies \& J.M. 
Campbell, under wet moldy bark on dead tree, (CNC, 23); St. Thomas, 28.VII.1925, James, (CNC, 1); Strathroy, 20.VI.1934, H.F. Hudson, (CNC, 1); Sudbury, 1889, (CNC, 1); Toronto, 20.XI.1895, R.J. Crew, (CUIC, 2); same locality, 24.VIII.1895, R.J. Crew, (CUIC, 5); same locality, 11.XI.1894, R.J. Crew, (CUIC, 1); same locality, 31.VIII.1895, R.J. Crew, (CUIC, 1); same locality, 24.XII.1894, R.J. Crew, (CUIC, 2); same locality, 7.X.1978, R.S. Anderson, (ROME, 2); Dundas, 16.V.1979, Dael Morris, (DEBU, 1); Gananoque, 6.VII.1963, C.J. Edwards, (DEBU, 1); Georgetown, 27.V.1988, R. Gagne, Malaise trap, (DEBU, 1); Guelph, 9.V.1975, J.M. Heraty, (DEBU, 4); same locality, 15.VII.1975, Sandra Allan, (DEBU, 1); same locality, 25.VII.1982, W. Punchihewa, (DEBU, 1); same locality, 17.VII.1976, S.A. Marshall, (DEBU, 1); same locality, 10.V.1975, J. Umphrey, (DEBU, 4); Haulock, 9.VII.1977, Mccreadie, (DEBU, 1); Kingsville, 8.VII.1977, A.A. Konecny, (DEBU, 1); Lanark, 2.VIII.1976, M.J. Sharkey, (DEBU, 1); London, W. Saunders, (DEBU, 1); Norval, 16.V.1980, Kevin Barber, (DEBU, 1); Toronto, 10.VII.1977, W.A. Attwater, (DEBU, 1); Vineland, 20.VIII.1930, W.L. Putman, (DEBU, 1). QUEBEC: Aylmer, Queen's Park, 24.X.1923, C.B. Hutchings, (CNC, 1); Chelsea, 25.VI.1912, A. Gibson, (CNC, 1); Deux-Montagnes, Parc Paul-Sauvé, 9.IV.1989, S. Laplante, (SLC, 1); Dorval, 26.IX.1985, LeSage \& Smetana, Ex. Pleurotus ostreatus in decid. forest, (CNC, 2); Gatineau, Eardley, 11.VI.1994, F. Genier, (CMNC, 1); Ile-de-Mtl, bois de Saraguay, 21.IV.1979, S. Laplante, (SLC, 3); Limbour Touraine, 12.VIII.1974, R. Sexton, (CNC, 1); Masham Twp., Gatineau Co., 27. VIII.1975, D.M. Wood, teneral, (CNC, 3); Meach Lk., Gatineau Park, 11.VIII.1976, J.M. Campbell, (CNC, 1); Missisquoi, le Petit Pinacle, 24.IV.1979, S. Laplante, (SLC, 1); Montreal, 24.VII.1970, E.J. Kiteley, (CNC, 1); same locality, 1.VII.1970, E.J. Kiteley,

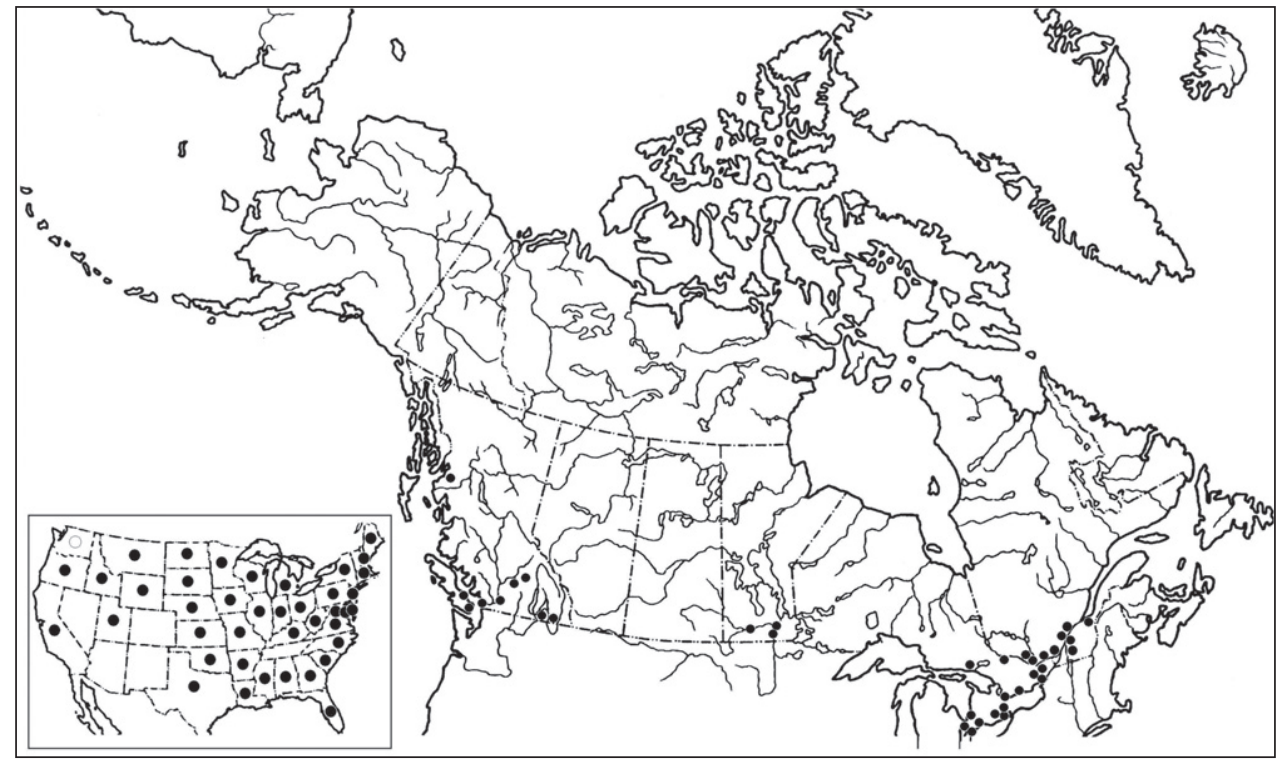

Fig. 3 I. Canadian distribution of Synstrophus repandus. Inset shows US distribution, by state. Open circle indicates state record from Hatch (1965). 
fungus on log, (CNC, 1); same locality, 8.VI.1976, E.J. Kiteley, on fungus, stump, (CNC, 4); same locality, 12.VI.1981, E.J. Kiteley, on fungus, stump, (CNC, 1); same locality, 28.VI.1982, E.J. Kiteley, UV light trap, (CNC, 1); same locality, 12.VIII.1972, E.J. Kiteley, on log fungus, (CNC, 2); same locality, 1.IX.1972, E.J. Kiteley, (CNC, 1); same locality, 11.VII.1982, E.J. Kiteley, UV light trap, (CNC, 1); same locality, (MCZ, 1); Pontiac, Eardley, 24.VIII.1991, S. Laplante, polypore on branch of old Quercus rubrus, beaten, (SLC, 1); Terrebonne, 17.VIII.1918, F. T. Scott, (LACM, 4); same locality, 17.VIII.1918, (UAIC, 2); Vaudreuil, Rigaud, 16.VI.1990, S. Laplante, (SLC, 2).

\section{Discussion}

The Eustrophinae, like many groups of "little brown beetles", are rather obscure, infrequently encountered by general collectors, and without much detailed knowledge of their biology. Their somewhat cryptic habits and habitats, and predominantly nocturnal activity have allowed this obscurity to continue. However, in preferred habitats (i.e. dead trees with wood-rotting fungi), individuals of some species (e.g. E. bicolor and S. repandus) can be very abundant. One must be willing to visit these habitats after dark, and examine dead wood surfaces and/or associated fungi.

Eustrophines are a small part of the entire group of saproxylic Coleoptera, which includes species that are wood and bark feeders, fungal feeders, and predators (Jonsell, 1999). In forest habitats, saproxylic Coleoptera represent a large component of the beetle fauna (Hammond et al., 2004; Siitonen, 2001). For example, about 25\% of Swedish beetles are saproxylic (Jonsell, 1999); these species are partly responsible for the breakdown of coarse woody material, through direct feeding action and transferring fungi among individual logs, snags and stumps (Hammond et al., 2004). Most studies on the importance of saproxylic Coleoptera to forest ecology and management were done in Europe, notably in Scandinavia (e.g. Martikainen, 2001; Siitonen, 2001). European Coleoptera are better known, both taxonomically and ecologically, with habitat associations generally well understood for most species. Studies on saproxylics in North America have lagged somewhat behind, although they have become better known through increased awareness of their importance in forest habitats in Canada and the United States. Indeed, according to Hammond et al. (2004: 16) "in North America, saproxylic assemblages are poorly understood and woefully understudied".

Forest management practices can significantly affect diversity and abundance of saproxylic Coleoptera (Majka and Pollock, 2006), although most considerations of the effects of forest practices focus on vertebrates (Hammond et al., 2004). Some recent studies in Canada (e.g. Hammond, 1997; Hammond et al., 2001; Majka and Pollock, 2006) have revealed a rich fauna of saproxylic Coleoptera, although there is much more to learn about these ecologically important beetles. Although the Canadian Eustrophinae are relatively few in species, they are occasionally very abundant in wood-rotting fungal habitats, and are undoubtedly an integral component of the "coarse woody debris" insect assemblage. As suggested by Majka and Pollock (2006), further work 
is required to initially identify the saproxylic beetle assemblages, determine their role in forest communities, and then to recommend silvicultural practices that minimize impact on these insects. For the Canadian eustrophines specifically, very little is known about their feeding preferences, life histories, immature stages and other bionomic parameters. Perhaps once these are better known, the ecological importance of these historically obscure beetles will be shown to be greater than previously thought.

\section{Acknowledgements}

Thanks to Lyubomir Penev and Chris Majka for suggesting that I submit a contribution to this special "Canadian" Zookeys issue. Mr. Dan Marschalek generously provided his time and expertise in taking the photographs shown in Figs 1-14. Studies like this one are not possible without specimens. I would like to thank the curators who gave me the privilege of examining material under their care. I have been very fortunate to have received assistance and specimens from many collectors over the years. Among them, the most memorable and influential were the late John and Bert Carr of Calgary Alberta, who, for a very educational week back in the mid 1980s, invited a neophyte coleopterist into their home. Each day, we visited a different part of southern Alberta and collected a variety of beetles. Later, we would retire to the Carr's "beetle room" where the catch would be identified, with the benefit of John's vast knowledge and the very impressive collection that both Bert and John had amassed. Later, the Carrs generously donated specimens to my collection (and others). Indeed, Carr-collected specimens form a significant part of this paper. I dedicate this paper to the memory of Alberta and John Carr, "amateur" Canadian beetle collectors extraordinaire.

\section{Literature}

Arnett R Jr (1968) The beetles of the United States (a manual for identification). The American Entomological Institute, Ann Arbor, 1112 pp.

Blackwelder R (1945) Checklist of the coleopterous insects of Mexico, Central America, the West Indies, and South America. Part 3. Bulletin of the United States National Museum 185: 343-550.

Blatchley W (1910) An Illustrated Descriptive Catalogue of the Coleoptera or Beetles (Exclusive of the Rhynchophora) Known to Occur in Indiana - With Bibliography and Descriptions of New Species. The Nature Publishing Co., Indianapolis, 1386 pp.

Champion G (1889) Coleoptera (Lagriidae, Melandryidae, Pythidae, Oedemeridae). In: Godman, FD, Salvin, O (Eds). Biologia Centrali-Americana, Insecta, Coleoptera, Volume 4, Part 1. Dulau, London,1-494.

Champion G (1915) Notes on Melandryidae. Entomologists Monthly Magazine 51: 138-140.

Champion G (1916) Notes on Melandryidae (3). Entomologists Monthly Magazine 2 (Ser. 3): 75-83, 99-108, 144-157 + plate II. 
Chantal C (1985) Les Tetratomidae (Coleoptera) du Québec. Fabreries 11(3): 43-66 + maps.

Crowson R (1964) Observations on British Tetratomidae (Col.) with a key to the larvae. Entomologists Monthly Magazine 99: 82-86.

Csiki E (1924) Pars 77. Serropalpidae. Coleopterorum Catalogus. Volume 17. W. Junk, Berlin.

Dury C (1906) Ecological notes on some Coleoptera of the Cincinnati region, including seven new species. Journal of the Cincinnati Society of Natural History 20(7): 251-256.

Fabricius J (1792) Entomologia systematica emendata et aucta. Secundum classes, ordines, genera, species adiectis synonymis, locis, observationibus, descriptionibus. Proft, Hafniae, 538 pp.

Fabricius J (1798) Supplementum entomologiae systematicae. Proft \& Storch, Hafniae, 572 pp. Gistel J (1856) Die Mysterien der Europäischen Insectenwelt. Dannheimer, Kempten, 12 + 532 pp. Hammond H (1997) Arthropod biodiversity from Populus coarse woody material in north-central Alberta: a review of taxa and collection methods. Canadian Entomologist 129: 1009-1033.

Hammond H, Langor D, Spence J (2001) Early colonization of Populus wood by saproxylic beetles. Canadian Journal of Forest Research 31: 1175-1183.

Hammond H, Langor D, Spence J (2004) Saproxylic beetles (Coleoptera) using Populus in boreal aspen stands of western Canada: spatiotemporal variation and conservation of assemblages. Canadian Journal of Forest Research 34: 1-19.

Hatch M (1965) The Beetles of the Pacific Northwest. Part IV: Macrodactyles, Palpicornes, and Heteromera. University of Washington Press, Seattle, 268 pp.

Hayashi N (1975) On the larvae of Melandryidae (Coleoptera, Cucujoidea) and some related families occurring in Japan. Kontyû 43(2): 147-169.

Horn G (1888) Miscellaneous coleopterous studies. Transactions of the American Entomological Society 15: 26-48.

Illiger J (1802) Aufzählung der Käfergattungen nach der Zahl der Fussglieder. Magazin für Insektenkunde 1: 285-305.

Jonsell M (1999) Insects in wood-decaying polypores: conservation aspects. Ph.D. thesis, University of Uppsala, Sweden.

Laporte [= Castelnau] FLNC de (1840) Histoire naturelle des insectes coléoptères. Tome deuxième. P. Duménil, Paris, 563 pp.

Lawrence J (1991) Melandryidae (Tenebrionoidea) (= Serropalpidae). In: Stehr FW (Ed) Immature Insects. Volume 2 (Coleoptera). Kendall/Hunt Publ. Co., Dubuque, 505-508.

Lawrence J, Newton A (1995) Families and subfamilies of Coleoptera (with selected genera, notes, references and data on family-group names). In: Pakaluk J, Ślipiński SA (Eds) Biology, Phylogeny, and Classification of Coleoptera: Papers Celebrating the $80^{\text {th }}$ Birthday of Roy A. Crowson. Muzeum i Instytut Zoologii PAN, Warszawa, 779-1092.

LeConte J (1866) List of the Coleoptera of North America. Part I. Smithsonian Miscellaneous Collections (6) 140: 1-78.

LeConte J (1873) Synonymical remarks upon North American Coleoptera. Proceedings of the Academy of Natural Sciences of Philadelphia 3: 321-336.

LeConte J (1874) Descriptions of new Coleoptera chiefly from the Pacific slope of North America. Transactions of the American Entomological Society 5: 43-72.

Leng C (1920) Catalogue of the Coleoptera of America, north of Mexico. John D. Sherman, Jr., Mount Vernon, 470 pp. 
Leng C, Mutchler A (1933) Second and third supplements 1925 to 1932 (inclusive) to Catalogue of the Coleoptera of America, north of Mexico. John D. Sherman, Jr., Mount Vernon, 112 pp.

LeSage L (1991) Family Melandryidae, false darkling beetles. In: Bousquet Y (Ed) Checklist of Beetles of Canada and Alaska. Agriculture Canada, Ottawa, 245-248.

Majka C, Pollock D (2006). Understanding saproxylic beetles: new records of Tetratomidae, Melandryidae, Synchroidae, and Scraptiidae from the Maritime Provinces of Canada (Coleoptera: Tenebrionoidea). Zootaxa 1248: 45-68.

Martikainen P (2001) Conservation of threatened saproxylic beetles: significance of retained aspen Populus tremula on clearcut areas. Ecological Bulletins 49: 205-218.

Melsheimer F (1846) Descriptions of new species of Coleoptera of the United States. Proceedings of the Academy of Natural Sciences of Philadelphia 3: 53-66.

Melsheimer F (1853) Catalogue of the described Coleoptera of the United States. Revised by S.S. Haldeman and J. L. LeConte. Washington, Smithsonian Institution, xvi + 174 pp.

Nikitsky N (1983) Un nouveau genre de la famille des melandryides (Coleoptera) de Chine. Bulletin de la Société Entomologique de Mulhouse 1983 (juillet-septembre): 37-38.

Nikitsky N (1998) Generic Classification of the Beetle Family Tetratomidae (Coleoptera, Tenebrionoidea) of the world, with description of new taxa. Pensoft Publishers, Sofia-Moscow, 80 pp.

Pic M (1954) Coléoptères nouveaux de Chine. Bulletin de la Société Entomologique de Mulhouse 1954 (sept.-oct.): 53-59.

Poole R, Gentili P (Eds) (1996) Fauna Insecta Nearctica. Vol. 1: Coleoptera, Strepsiptera. Entomological Information Services, Rockville, $827 \mathrm{pp}$.

Provancher L (1877) Petite Faune Entomologique du Canada, precedée d'un Traite Elementaire d'Entomologie. Volume I - les Coléoptères. Darveau, Quebec City, 785 pp + errata.

Reitter E (1887) [title of article unknown]. Deutsche Entomologische Zeitschrift 31: 383.

Say T (1824) Class Insecta. In: Keating WH (Ed) Narrative of an expedition to the source of St. Peter's River, Lake Winnepeek, Lake of the Woods, performed in the year 1823, by order of the Hon. J.C. Calhoun, Secretary of War, under the command of Stephen H. Long, Major U.S.T.E. Vol. II. Carey \& Lea, Philadelphia, 268-378.

Say T (1826) Descriptions of new species of coleopterous insects inhabiting the United States (continued). Journal of the Academy of Natural Sciences of Philadelphia 5: 237-284.

Seidlitz G von (1898) Melandryidae. In: Erichson WF, Schaum H, Kraatz G, Kiesenwetter H von, Weise J, Reitter E, Seidlitz G (Eds) Naturgeschichte der Insecten Deutschlands. Erste Abtheilung Coleoptera, Fünfter Band, Zweite Hälfte, Lieferungen 1-3. Nicolaische Verlags-Buchhandlung, Berlin, 365-380.

Siitonen J (2001) Forest management, coarse woody debris and saproxylic organisms: Fennoscandian boreal forests as an example. Ecological Bulletins 49: 11-41.

Viedma M de (1971) Redescripcion de la larva de Eustrophinus bicolor y consideraciones acerca de la position sistematica del genero Eustrophinus (Col. Melandryidae). Annales de la Société Entomologique de France (N.S.) 7 (3): 729-733.

Weiss H (1919) Notes on Eustrophus bicolor Fabr., bred from fungi (Coleoptera). Psyche 26: 132-133. Young D, Pollock D (2002) Family 99. Tetratomidae. In: Arnett RH Jr, Thomas M, Skelley P, Frank J. (Eds). American Beetles. Volume 2. Polyphaga: Scarabaeoidea through Curculionoidea. CRC Press, Boca Raton. 413-416. 


\section{Checklist of Nearctic species of Eustrophinae (classification follows Nikitsky 1998)}

Eustrophinae Gistel, 1856

Tribe Holostrophini Nikitsky, 1998

Pseudoholostrophus Nikitsky, 1983

P. (Holostrophinus) discolor (Horn, 1888)

P. (Pseudoholostrophus) impressicollis (LeConte, 1874)

Holostrophus Horn, 1888

H. bifasciatus (Say, 1824)

Tribe Eustrophini Gistel, 1856

Eustrophus Illiger, 1802

E. tomentosus Say, 1826

Synstrophus Seidlitz, 1898

S. repandus (Horn, 1888)

Eustrophopsis Champion, 1889 (= Eustrophinus Seidlitz, 1898)

E. arizonensis (Horn, 1888)

E. bicolor (Fabricius, 1798)

E. brunneimarginatus (Dury, 1906)

E. confinis (LeConte, 1866)

E. indistinctus (LeConte, 1851)

E. ornatus (Van Dyke, 1928) 